J. DIFFERENTIAL GEOMETRY

73 (2006) 359-412

\title{
HAMILTONIAN 2-FORMS IN KÄHLER GEOMETRY, I GENERAL THEORY
}

\author{
Vestislav Apostolov, David M.J. Calderbank \\ \& Paul Gauduchon
}

\begin{abstract}
We introduce the notion of a hamiltonian 2-form on a Kähler manifold and obtain a complete local classification. This notion appears to play a pivotal role in several aspects of Kähler geometry. In particular, on any Kähler manifold with co-closed Bochner tensor, the (suitably normalized) Ricci form is hamiltonian, and this leads to an explicit description of these Kähler metrics, which we call weakly Bochner-flat. Hamiltonian 2-forms also arise on conformally Einstein Kähler manifolds and provide an Ansatz for extremal Kähler metrics unifying and extending many previous constructions.
\end{abstract}

In a previous paper [3], while investigating Kähler 4-manifolds whose antiselfdual Weyl tensor is co-closed, we happened upon a remarkable linear differential equation for $(1,1)$-forms $\phi$ on a Kähler manifold. This equation states (in any dimension)

$$
\nabla_{X} \phi=\frac{1}{2}\left(d \operatorname{tr}_{\omega} \phi \wedge g(J X, \cdot)-J d \operatorname{tr}_{\omega} \phi \wedge g(X, \cdot)\right)
$$

for all vector fields $X$, where $(g, J, \omega)$ is the Kähler structure with LeviCivita connection $\nabla$. A hamiltonian 2-form is a (nontrivial) solution $\phi$ of (1).

Hamiltonian 2-forms underpin many explicit constructions in Kähler geometry. They arise in particular on Bochner-flat Kähler manifolds and on Kähler manifolds (of dimension greater than four) which are conformally Einstein, both of which have been classified recently, respectively by Bryant [8], and Derdziński and Maschler [11]. In this paper we obtain an explicit local classification of all Kähler metrics with a hamiltonian 2-form, which provides a unifying framework for these works, and at the same time extends Bryant's local classification to the

The first author was supported in part by NSERC grant OGP0023879, the second author by the Leverhulme Trust and the William Gordon Seggie Brown Trust. All three authors are members of EDGE, Research Training Network HPRN-CT-200000101, supported by the European Human Potential Programme.

Received 03/06/2004. 
much larger class of Kähler manifolds with co-closed Bochner tensor, called weakly Bochner-flat.

The key feature of hamiltonian 2-forms $\phi$ on Kähler $2 m$-manifolds $M$ - and the reason for the name - is that if $\sigma_{1}, \ldots \sigma_{m}$ are the elementary symmetric functions of the $m$ eigenvalues of $\phi$ (viewed as a hermitian operator via the Kähler form $\omega$ ), then the hamiltonian vector fields $K_{r}=J \operatorname{grad}_{g} \sigma_{r}$ are Killing. Further, the Poisson brackets $\left\{\sigma_{r}, \sigma_{s}\right\}$ are all zero, so that the vector fields $K_{1}, \ldots K_{m}$ commute.

If $K_{1}, \ldots K_{m}$ are linearly independent, then the Kähler metric is toric. However, not every toric Kähler metric arises in this way: the hamiltonian property also implies that the eigenvalues of $\phi$ have orthogonal gradients. We say that a toric manifold is orthotoric if there is a momentum map $\left(\sigma_{1}, \ldots \sigma_{m}\right)$ for the torus action (with respect to some basis of the Lie algebra) such that the gradients of the roots of the polynomial $\sum_{r=0}^{m}(-1)^{r} \sigma_{r} t^{m-r}$ are orthogonal-here $\sigma_{0}=1$.

Of course $K_{1}, \ldots K_{m}$ need not be independent; then on an open set where the span is $\ell$-dimensional, there is a local hamiltonian $\ell$-torus action by isometries, so the Kähler metric on $M$ may be described (locally) by the Pedersen-Poon construction [22], as a fibration, with $2 \ell$ dimensional toric fibres, over a $2(m-\ell)$-dimensional complex manifold $S$ equipped with a family of Kähler quotient metrics parameterized by the momentum map of the local $\ell$-torus action.

The hamiltonian property of $\phi$ has further implications for the geometry of this fibration and of the base $S$. We say that a hamiltonian $\ell$-torus action is rigid if the metric on the orbits depends only on the momentum map, and that the base $S$ is semisimple if the Kähler quotient metrics are simultaneously diagonalizable and have common Levi-Civita connection. The rigidity condition has its origins in work of Calabi on Kähler metrics on holomorphic bundles $[\mathbf{9}, \mathbf{1 0}]$ and has a number of formulations: it means, for instance, that the local fibration of $M$ over $S$ is totally geodesic, or equivalently, that $M$ is associated, locally, to a principal $\ell$-torus bundle with connection over $S$. When $\ell=1$ semisimplicity is closely related to the ' $\sigma$-constancy' of Hwang-Singer [15]. Both rigidity and semisimplicity have explicit descriptions as special cases of the Pedersen-Poon construction [22].

Our main result shows that any Kähler manifold with a hamiltonian 2 -form induces a (local) semisimple rigid $\ell$-torus action $(\ell \leq m)$ with orthotoric fibres, and that conversely, such an explicit Kähler metric has a hamiltonian 2-form. From this we deduce a local classification of weakly Bochner-flat Kähler metrics, rederiving in particular Bryant's classification of Bochner-flat Kähler metrics. We also obtain a new proof of the Derdziński-Maschler classification of conformally Einstein Kähler metrics in higher dimensions, and an Ansatz for extremal Kähler metrics - including all constant scalar curvature and Kähler-Einstein 
metrics with a hamiltonian 2-form - which unifies and extends many constructions in the literature.

The structure of the paper is as follows. In section 1, after reviewing some background material, we explain how equation (1) arises on weakly Bochner-flat Kähler manifolds and on conformally Einstein Kähler manifolds. Thus motivated, we begin the study of hamiltonian 2-forms in section 2, where we derive the existence of the hamiltonian Killing vector fields, and show that the equation for hamiltonian 2-forms is an overdetermined equation of finite type which is completely integrable on manifolds of constant holomorphic sectional curvature.

In section 3 we study (isometric) hamiltonian torus actions in general. This section is almost entirely independent of the first two, although our analysis is motivated by the special properties of hamiltonian 2-forms. We first show that the Pedersen-Poon construction $[\mathbf{2 2}]$ has a natural and essentially coordinate-free description in terms of a potential $G$, which is a fibrewise Legendre transform of a Kähler potential, and is required to satisfy only open conditions - such 'dual potentials' appeared first in the toric case, in work of Guillemin [14] and Abreu [1]. We also describe the invariant pluriharmonic functions and compute the Ricci form.

In subsections 3.2 and 3.3 we introduce rigid and semisimple hamiltonian torus actions respectively. In the case of circle actions (in particular) these conditions originally arose as an Ansatz for the construction of extremal Kähler metrics and Kähler-Einstein metrics $[\mathbf{9}, \mathbf{1 0}, \mathbf{1 5}, \mathbf{1 7}$, 22, 25, 26, 27]: for semisimple rigid actions in momentum coordinates, the Ricci form is linear in the matrix of inner products of the Killing vector fields generating the action. Our approach provides a natural interpretation, particularly for the rigidity condition.

Subsection 3.4 is devoted to orthotoric Kähler metrics: in four dimensions, these were introduced in $[\mathbf{3}]$ and explicitly classified; here we extend the definition and classification to all dimensions. The decisive feature of orthotoric $2 m$-manifolds is that they depend effectively on $m$ functions of 1 variable, rather than the 1 function of $m$ variables (the dual potential $G$ ) that governs toric Kähler metrics in general. This means that curvature conditions are (functional) ordinary differential equations, rather than partial differential equations.

The central results of this paper can be found in section 4, where we bring the work of sections 2 and 3 together. We first prove that on a connected Kähler $2 m$-manifold with hamiltonian 2-form $\phi$ and associated Killing vector fields $K_{1}, \ldots K_{m}$, there is an integer $\ell$, with $0 \leq \ell \leq m$, such that the span of $K_{1}, \ldots K_{m}$ is everywhere at most $\ell$ dimensional, but on a dense open set $K_{1}, \ldots K_{\ell}$ are linearly independent. We show that $\ell$ roots of the momentum polynomial $p(t):=(-1)^{m} \operatorname{pf}(\phi-$ $t \omega)$ are functionally independent, the remainder being constant. (Here 
pf $\psi=\frac{1}{m !} *\left(\psi^{\wedge m}\right)$ stands for the pfaffian of a 2 -form $\psi$.) We call $\ell$ the order of $\phi$ and prove that Kähler manifolds admitting a hamiltonian 2form of order $\ell$ are exactly those admitting a local hamiltonian $\ell$-torus action such that

- the fibres are orthotoric;

- the action is rigid;

- the base is semisimple, with relative eigenvalues of a special form.

In section 5 we study the curvature of our explicit metrics and hence obtain classifications of extremal Kähler metrics with a hamiltonian 2form, of weakly Bochner-flat Kähler metrics, and of Bochner-flat Kähler metrics [8].

To summarize, we have the following result.

Theorem. Let $(M, g, J, \omega)$ be a connected Kähler $2 m$-manifold with a hamiltonian 2-form $\phi$ of order $\ell$. Then there are functions $F_{1}, \ldots F_{\ell}$ of one variable such that on a dense open subset $M^{0}$ of $M$, the Kähler structure may be written

$$
\begin{aligned}
g & =\sum_{\xi} p_{\mathrm{nc}}(\xi) g_{\xi}+\sum_{j=1}^{\ell} \frac{p^{\prime}\left(\xi_{j}\right)}{F_{j}\left(\xi_{j}\right)} d \xi_{j}^{2}+\sum_{j=1}^{\ell} \frac{F_{j}\left(\xi_{j}\right)}{p^{\prime}\left(\xi_{j}\right)}\left(\sum_{r=1}^{\ell} \sigma_{r-1}\left(\hat{\xi}_{j}\right) \theta_{r}\right)^{2}, \\
\omega & =\sum_{\xi} p_{\mathrm{nc}}(\xi) \omega_{\xi}+\sum_{r=1}^{\ell} d \sigma_{r} \wedge \theta_{r}, \quad d \theta_{r}=\sum_{\xi}(-1)^{r} \xi^{\ell-r} \omega_{\xi}, \\
J d \xi_{j} & =\frac{F_{j}\left(\xi_{j}\right)}{p^{\prime}\left(\xi_{j}\right)} \sum_{r=1}^{\ell} \sigma_{r-1}\left(\hat{\xi}_{j}\right) \theta_{r}, \quad J \theta_{r}=(-1)^{r} \sum_{j=1}^{\ell} \frac{p_{\mathrm{c}}\left(\xi_{j}\right)}{F_{j}\left(\xi_{j}\right)} \xi_{j}^{\ell-r} d \xi_{j} .
\end{aligned}
$$

Any Kähler metric of this form admits a hamiltonian 2-form of order $\ell$, namely

$$
\phi=\sum_{\xi} \xi p_{\mathrm{nc}}(\xi) \omega_{\xi}+\sum_{r=1}^{\ell}\left(\sigma_{r} d \sigma_{1}-d \sigma_{r+1}\right) \wedge \theta_{r} .
$$

In these expressions:

- $\sigma_{r}$ is the rth elementary symmetric function of the non-constant roots $\xi_{1}, \ldots \xi_{\ell}$ of the momentum polynomial $p(t)-$ so $\sigma_{\ell+1}=0$ - and $\sigma_{r-1}\left(\hat{\xi}_{j}\right)$ is the $(r-1)$ st elementary symmetric functions of the $\ell-1$ roots $\left\{\xi_{k}: k \neq j\right\}$

- summation over $\xi$ denotes the sum over the different constant roots of the momentum polynomial and $\left(g_{\xi}, \omega_{\xi}\right)$ is a positive or negative definite Kähler metric on a manifold $S_{\xi}$ of the same (real) dimension $2 m_{\xi}$ as the $\xi$-eigenspace of $-J \circ \phi$;

- $p(t)=p_{\mathrm{nc}}(t) p_{\mathrm{c}}(t)$, where $p_{\mathrm{nc}}(t)=\prod_{j=1}^{\ell}\left(t-\xi_{j}\right)$ and $p_{\mathrm{c}}(t)=$ $\prod_{\xi}(t-\xi)^{m_{\xi}}$; also $p^{\prime}(t)$ is the $t$-derivative of $p(t)$, so that $p^{\prime}\left(\xi_{j}\right)=$ $p_{\mathrm{c}}\left(\xi_{j}\right) \prod_{k \neq j}\left(\xi_{j}-\xi_{k}\right)$. 
Now define polynomials $\check{p}_{\mathrm{c}}(t)=\prod_{\xi}(t-\xi)^{m_{\xi}-1}$ and $\hat{p}_{\mathrm{c}}(t)=\prod_{\xi}(t-$ $\xi)^{m_{\xi}+1}$. Then we have the following special cases:

(i) $(g, J, \omega)$ is an extremal Kähler metric if

- for all $j, F_{j}^{\prime \prime}(t)=\check{p_{\mathrm{c}}}(t)\left(\sum_{r=0}^{\check{m}} a_{r} t^{\check{m}-r}\right)$, where $a_{0}, \ldots a_{\check{m}}$ are arbitrary constants (independent of $j$ ) and $\check{m}=\ell+\sum_{\xi} 1=m-\sum_{\xi}\left(m_{\xi}-1\right)$;

- for all $\xi, \pm\left(g_{\xi}, \omega_{\xi}\right)$ has $\mathrm{Scal}_{ \pm g_{\xi}}=\mp\left(\sum_{r=0}^{\check{m}} a_{r} \xi^{\check{m}-r}\right) / \prod_{\eta \neq \xi}(\xi-\eta)$.

The scalar curvature $\mathrm{Scal}_{g}$ is constant if and only if $a_{0}=0$ and zero if and only if also $a_{1}=0$. An extremal Kähler metric with a hamiltonian 2 -form of order $\ell>0$ arises in this way if $\operatorname{grad}_{g} \mathrm{Scal}_{g}$ is tangent to the fibration defined by the 2-form.

(ii) $(g, J, \omega)$ is weakly Bochner-flat if

- for all $j, F_{j}^{\prime}(t)=p_{\mathrm{c}}(t)\left(\sum_{r=-1}^{\ell} b_{r} t^{\ell-r}\right)$, where $b_{-1}, \ldots b_{\ell}$ are arbitrary constants (independent of $j$ );

- for all $\xi, \pm\left(g_{\xi}, \omega_{\xi}\right)$ is Kähler-Einstein with 'Kähler-Einstein constant'

$$
\frac{1}{m_{\xi}} \mathrm{Scal}_{ \pm g_{\xi}}=\mp \sum_{r=-1}^{\ell} b_{r} \xi^{\ell-r}
$$

$(g, J, \omega)$ is Kähler-Einstein if and only if $b_{-1}=0$ and Ricci-flat if and only if also $b_{0}=0$. Any weakly Bochner-flat Kähler metric arises in this way.

(iii) $(g, J, \omega)$ is Bochner-flat if

- for all $j, F_{j}(t)=\hat{p}_{\mathrm{c}}(t)\left(\sum_{r=-2}^{\hat{m}} c_{r} t^{\hat{m}-r}\right)$, where $c_{-2}, \ldots c_{\hat{m}}$ are arbitrary constants (independent of $j$ ) and $\hat{m}=\ell-\sum_{\xi} 1=m-\sum_{\xi}\left(m_{\xi}+\right.$ $1)$

- for all $\xi, \pm\left(g_{\xi}, \omega_{\xi}\right)$ has constant holomorphic sectional curvature

$$
\frac{1}{m_{\xi}\left(m_{\xi}+1\right)} \mathrm{Scal}_{ \pm g_{\xi}}=\mp\left(\sum_{r=-2}^{\hat{m}} c_{r} \xi^{\hat{m}-r}\right) \prod_{\eta \neq \xi}(\xi-\eta) .
$$

$(g, J, \omega)$ has constant holomorphic sectional curvature if and only if $c_{-2}=0$ and is flat if and only if also $c_{-1}=0$. Any Bochner-flat Kähler metric arises in this way.

This theorem follows from Theorems 1 and 2, and Propositions 15, 16 and 17 in the text below. We end by discussing hamiltonian 2-forms of order 1, and the classification of conformally Einstein Kähler metrics $[\mathbf{1 1}]$. There are also two appendices. In Appendix A, we relate hamiltonian 2-forms to conformal Killing forms, recently studied by Moroianu and Semmelmann $[\mathbf{2 0}, \mathbf{2 4}]$. In Appendix B, we collect some Vandermonde identities, which we have used freely in the paper.

We thank Uwe Semmelmann for discussing conformal Killing forms with us, and Christina Tønnesen-Friedman for her helpful comments and interest in this work. 


\section{The curvature of a Kähler manifold}

In this section we review some background material in order to fix notation, and to present the notions of Bochner-flat, weakly Bochnerflat, and conformally Einstein Kähler metrics. Our conventions mainly follow $[7]$.

1.1. Riemannian curvature. The curvature $R$ of a $n$-dimensional riemannian manifold $(M, g)$ is defined by

$$
R_{X, Y} Z=\nabla_{[X, Y]} Z-\left[\nabla_{X}, \nabla_{Y}\right] Z
$$

for all vector fields $X, Y, Z$, where $\nabla$ denotes the Levi-Civita connection. It is a 2 -form with values in the adjoint bundle $A M$ (the bundle of skew endomorphisms of the tangent bundle $T M$ ) and satisfies the algebraic Bianchi identity: $R_{X, Y} Z+R_{Y, Z} X+R_{Z, X} Y=0$. Via the metric $g, A M$ can be identified with the bundle $\Lambda^{2} M$ of 2 -forms and $R$ can be viewed as a section of $\Lambda^{2} M \otimes \Lambda^{2} M$. Then, the algebraic Bianchi identity is equivalent to the following two conditions:

(i) $R$ belongs the the symmetric part, $S^{2} \Lambda^{2} M$, of $\Lambda^{2} M \otimes \Lambda^{2} M$;

(ii) $R$ belongs to the kernel of the linear map, $\beta$, from $S^{2} \Lambda^{2} M$ to $\Lambda^{4} M$ determined by the wedge product.

$\mathcal{R} M:=\operatorname{ker} \beta \subseteq S^{2} \Lambda^{2} M$ is called the bundle of (abstract) curvature tensors.

The Ricci contraction is the linear map $c$ from $\mathcal{R} M$ to the bundle $S M$ of symmetric bilinear forms of $M$ sending $R$ to the bilinear form Ric defined by $\operatorname{Ric}_{X, Y}=\operatorname{tr}\left(Z \rightarrow R_{X, Z} Y\right)$. We thus obtain an orthogonal decomposition:

$$
\mathcal{R} M=c^{*}(S M) \oplus \mathcal{W} M,
$$

where $\mathcal{W} M$, called the bundle of (abstract) Weyl tensors of $(M, g)$, denotes the kernel of $c$ in $\mathcal{R} M$. Accordingly, the curvature $R$ splits as $R=c^{*}(h)+W$, where $W$ is the Weyl tensor of $(M, g)$, whereas $h$ satisfies $c c^{*}(h)=$ Ric. For $n \geq 3, c$ is surjective; its adjoint $c^{*}$ is then injective and $h$ is determined by

$$
h=\frac{\text { Scal }}{2 n(n-1)} g+\frac{\mathrm{Ric}_{0}}{n-2},
$$

where Scal is the scalar curvature of $g$, i.e., the trace of Ric with respect to $g$, and $\mathrm{Ric}_{0}$ denotes the traceless part of Ric (so that Ric $=\frac{1}{n} \mathrm{Scal} g+$ Ric $_{0}$ ), which is a section of $S_{0} M$, the bundle of symmetric traceless bilinear forms. For $n=2, c^{*}$ has kernel $S_{0} M$ so that $\mathrm{Ric}_{0}=0$ and the tracefree part of $h$ is undetermined.

Finally, the curvature $R$, viewed as a symmetric endomorphism of $\Lambda^{2} M$ using $g$, splits into three orthogonal pieces as follows:

$$
R=\left.\frac{\text { Scal }}{n(n-1)} \mathrm{Id}\right|_{\Lambda^{2} M}+\frac{1}{n-2}\left\{\operatorname{Ric}_{0}, \cdot\right\}+W
$$


where $\left\{\operatorname{Ric}_{0}, \cdot\right\}$ acts on $\psi \in \Lambda^{2} M$ to give the anticommutator $\left\{\operatorname{Ric}_{0}, \psi\right\}$ $:=\operatorname{Ric}_{0} \circ \psi+\psi \circ \operatorname{Ric}_{0}$ of $\operatorname{Ric}_{0}$ and $\psi$, which are viewed, via $g$, as endomorphisms, respectively symmetric and skew, of $T M$. When $n=2$, the second term is zero.

Each piece of (2) is an element of $\mathcal{R} M$. The corresponding subbundle of $\mathcal{R} M$ is associated to an irreducible representation of the orthogonal group $O(n)$, respectively the trivial representation, the Cartan product $\mathbb{R}^{n} \odot \mathbb{R}^{n}$, the Cartan product $\mathfrak{o}(n) \odot \mathfrak{o}(n)$, where $\mathfrak{o}(n) \cong \Lambda^{2} \mathbb{R}^{n}$ denotes the Lie algebra of $O(n)$. (The Cartan product of two irreducible representations with dominant weights $\lambda_{1}$ and $\lambda_{2}$ is the irreducible subrepresentation of the tensor product with dominant weight $\lambda_{1}+\lambda_{2}$.)

1.2. The Bochner tensor of a Kähler manifold. Let $(M, g, J, \omega)$ be a Kähler manifold of dimension $n=2 m$. By definition, $J$ is an orthogonal complex structure that is parallel with respect to the Levi-Civita connection $\nabla$. The Kähler form $\omega$ is defined by $\omega(X, Y)=g(J X, Y)$. The Ricci form $\rho$ and its primitive part $\rho_{0}$ are defined in a similar way: $\rho(X, Y)=\operatorname{Ric}(J X, Y)$ and $\rho_{0}(X, Y)=\operatorname{Ric}_{0}(J X, Y)$. The Ricci tensor is $J$-invariant, and so $\rho$ and $\rho_{0}$ are 2 -forms. We denote by

$$
\Lambda^{2} M=\Lambda^{J,+} M \oplus \Lambda^{J,-} M
$$

the (orthogonal) decomposition of $\Lambda^{2} M$ into its $J$-invariant part, $\Lambda^{J,+} M$, and its $J$-anti-invariant part, $\Lambda^{J,-} M$. The riemannian curvature $R$ has values in $\Lambda^{J,+} M$ and therefore acts trivially on $\Lambda^{J,-} M$. More generally, we call an element of $\mathcal{R} M$ kählerian if it acts trivially on $\Lambda^{J,-} M$. The set of abstract kählerian curvature tensors is a vector subbundle of $\mathcal{R} M$, denoted by $\mathcal{K} M$; thus $\mathcal{K} M$ is the kernel of the linear map from $S^{2} \Lambda^{J,+} M$ to $\Lambda^{4} M$ determined by the wedge product.

The curvature tensor $R$ of a Kähler manifold $(M, g, J)$ is a section of $\mathcal{K} M$, but in general none of its components in (2) are. Indeed, the first component of $R$ in (2) is only an element of $\mathcal{K} M$ if it is zero or $n=2$, while the second component is only an element of $\mathcal{K} M$ if it is zero or $n=4$. We define the Bochner tensor $W^{\mathcal{K}}$ to be the orthogonal projection of the third component, the Weyl tensor $W$, onto $\mathcal{W} M \cap \mathcal{K} M$. We thus obtain a new decomposition of the curvature $R$ inside $\mathcal{K} M$.

$$
\begin{aligned}
R= & \frac{\text { Scal }}{2 m(m+1)}\left(\left.\mathrm{Id}\right|_{\Lambda^{J,+} M}+\omega \otimes \omega\right) \\
& +\frac{1}{m+2}\left(\left.\left\{\operatorname{Ric}_{0}, \cdot\right\}\right|_{\Lambda^{J,+} M}+\rho_{0} \otimes \omega+\omega \otimes \rho_{0}\right) \\
& +W^{\mathcal{K}} .
\end{aligned}
$$

Here $\left.\right|_{\Lambda^{J,+} M}$ has to be interpreted as the orthogonal projection $\psi \mapsto \psi^{J,+}$ of $\Lambda^{2} M$ onto its $J$-invariant part $\Lambda^{J,+} M$, and $\rho_{0} \otimes \omega$ acts on $\psi \in \Lambda^{2} M$ to give $\left\langle\rho_{0}, \psi\right\rangle \omega$, where the inner product on 2 -forms is normalized so that $\langle\omega, \omega\rangle=m=n / 2$. 
The three pieces of $R$ appearing in (3) are sections of subbundles of $\mathcal{K} M$ associated to irreducible representations of the unitary group $U(m)$, viewed as a subgroup of $O(n)$; namely: the trivial representation, the Cartan product $\mathbb{C}^{m} \odot \mathbb{C}^{m}$, and the Cartan product $\mathfrak{s u}(m) \odot \mathfrak{s u}(m)$ respectively, $\mathfrak{s u}(m)$ being the Lie algebra of $S U(m)$.

If Scal is a positive constant, then the first component of $R$ in (3) agrees with the curvature of the complex projective space $\mathbb{C} P^{m}$ with the Fubini-Study metric of holomorphic sectional curvature equal to $\frac{\text { Scal }}{m(m+1)}$.

The second component in (3) agrees with the second component in (2) when $n=4$, since then $\left\{\operatorname{Ric}_{0}, \psi^{J,-}\right\}=\left[J \psi^{J,-}, \rho_{0}\right]=0$, whereas $\left\{\operatorname{Ric}_{0}, \psi^{J,+}\right\}=\left\langle\psi, \rho_{0}\right\rangle \omega+\langle\psi, \omega\rangle \rho_{0}$. The four dimensional case is also special because the Weyl tensor $W$ splits into selfdual and antiselfdual parts as $W=W^{+}+W^{-}$, and on a Kähler 4-manifold the selfdual part is identified with the scalar curvature by

$$
W^{+}=\frac{\text { Scal }}{12}\left(\frac{3}{2} \omega \otimes \omega-\left.\mathrm{Id}\right|_{\Lambda^{J,+} M}\right)
$$

Bringing together $W^{+}$and the scalar part of $R$ in (2), we deduce that $W^{\mathcal{K}}=W^{-}$.

In higher dimensions, the Weyl tensor $W$ of a Kähler manifold splits into three pieces: one is the Bochner tensor $W^{\mathcal{K}}$, while the other two are identified with $\mathrm{Ric}_{0}$ and Scal. In other words, on a Kähler manifold of dimension $n \geq 6$ the information given by the riemannian curvature is already contained in the Weyl tensor; in particular, for $n \geq 6$, a locally conformally flat Kähler metric is flat.

1.3. The differential Bianchi identity in Kähler geometry. The differential Bianchi identity

$$
\nabla_{X} R_{Y, Z}+\nabla_{Y} R_{Z, X}+\nabla_{Z} R_{X, Y}=0
$$

easily implies the following one, known as the Matsushima identity:

$$
(\delta R)_{J X}=-\nabla_{X} \rho .
$$

(We specialize (4) by $X=e_{j}, Y=J e_{j}$, where $\left\{e_{j}\right\}$ is a local, $J$-adapted, orthonormal frame, and we observe that $\rho=\frac{1}{2} \sum_{j=1}^{n} R_{e_{j}, J e_{j}}$; here we define $\left.(\delta R)_{J X}:=-\sum_{j=1}^{n} \nabla_{e_{i}} R_{e_{i}, J X}.\right)$ The Matsushima identity immediately implies that the Ricci tensor of a Kähler manifold is parallel if and only if the curvature is co-closed, as a 2 -form with values in $\Lambda^{2} M$. The Ricci form $\rho$ may also be expressed as $\rho(X, Y)=\frac{1}{2} \sum_{j=1}^{n}\left\langle R_{X, Y} e_{j}, J e_{j}\right\rangle$, and so it is closed by (4). Hence from (3) and (5), we infer the following 
expression for the codifferential of the Bochner tensor:

$$
\begin{aligned}
\left(\delta W^{\mathcal{K}}\right)_{J X}= & -\frac{m}{m+2} \nabla_{X} \rho_{0}-\frac{1}{2(m+1)(m+2)} d \operatorname{Scal}(X) \omega \\
& +\frac{m}{4(m+1)(m+2)}\left(d \operatorname{Scal} \wedge J X-d^{c} \operatorname{Scal} \wedge X\right) .
\end{aligned}
$$

Here $d^{c}=J \circ d, X$ is any vector field, and we identify vector fields and 1 -forms via $g$. In view of this identity, we introduce a normalized Ricci form $\tilde{\rho}$ defined by

$$
\tilde{\rho}=\rho_{0}+\frac{\text { Scal }}{2 m(m+1)} \omega .
$$

Then, identity (6) reduces to

$$
\frac{m+2}{m}\left(\delta W^{\mathcal{K}}\right)_{J X}=-\nabla_{X} \tilde{\rho}+\frac{1}{2}\left(d s \wedge J X-d^{c} s \wedge X\right),
$$

where the normalized scalar curvature $s=\frac{\text { Scal }}{2(m+1)}$ is the trace of $\tilde{\rho}$ with respect to $\omega: s=\langle\tilde{\rho}, \omega\rangle$.

Definition 1. A Kähler manifold $(M, g, J)$ is called Bochner-flat (or Bochner-Kähler) if the Bochner tensor vanishes, $W^{\mathcal{K}}=0$, and weakly Bochner-flat if the Bochner tensor is co-closed, $\delta W^{\mathcal{K}}=0$.

By (7), a Kähler manifold is weakly Bochner-flat if and only if it satisfies the following weak Einstein condition:

$$
\nabla_{X} \tilde{\rho}=\frac{1}{2}\left(d s \wedge J X-d^{c} s \wedge X\right) .
$$

1.4. Conformally Einstein Kähler metrics. A Kähler manifold $(M, g, J, \omega)$ of dimension $n=2 m \geq 4$ is said to be conformally Einstein if there is a nonvanishing function $\tau$ such that $\tilde{g}:=\tau^{-2} g$ is an Einstein metric, i.e., $\operatorname{Ric}_{0}^{\tilde{g}}=0$. A straightforward and standard computation of the conformal change of the Ricci tensor shows that $g$ is conformally Einstein with conformal factor $\tau$ if and only if

$$
2(m-1) \nabla J d \tau=-\tau \rho+\lambda \omega
$$

for some function $\lambda$ - the trace of this equation then determines that

$$
\lambda=-\frac{m-1}{m} \Delta \tau+\frac{1}{2 m} \operatorname{Scal} \tau,
$$

where $\Delta \tau=-\operatorname{tr}_{g} \nabla d \tau=-\left\langle d d^{c} \tau, \omega\right\rangle$.

We recall that a hamiltonian vector field $K=J \operatorname{grad}_{g} f$ is Killing if and only if it preserves $J$, if and only if the hessian $\nabla d f$ is $J$-invariant, if and only if $\nabla J d f=\frac{1}{2} d d^{c} f$, in which case $f$ is said to be a Killing potential.

Clearly equation (9) implies that $2(m-1) \nabla J d \tau=(m-1) d d^{c} \tau$, so that by differentiating (9), we obtain $d \tau \wedge \rho-d \lambda \wedge \omega=0$ and hence $d \tau \wedge d \lambda \wedge \omega=0$. We shall say that $g$ is strongly conformally Einstein if 
$d \tau \wedge d \lambda=0$; this is automatic if $n \geq 6$ since the wedge product with $\omega$ is then injective on 2 -forms.

The fact that conformally Einstein Kähler metrics are strongly conformally Einstein in 6 or more dimensions was first observed by Derdziński and Maschler [11], who used this to obtain an explicit description of such metrics. A key step is essentially equivalent to the following.

Lemma 1 ([11]). Suppose that $g$ is strongly conformally Einstein, with conformal factor $\tau$. Then on the open set where $d \tau$ is nonzero,

$$
2 \nabla J d \tau=p \omega+q d \tau \wedge d^{c} \tau
$$

for some functions $p, q$ with $d p \wedge d \tau=0$.

Proof. On the open set where $d \tau$ is nonzero, we may write $d \lambda=\lambda_{\tau} d \tau$, so that $d \tau \wedge\left(\rho-\lambda_{\tau} \omega\right)=0$. It follows that the $J$-invariant 2-form $\rho-\lambda_{\tau} \omega$ is equal to $f d \tau \wedge d^{c} \tau$ for some function $f$. Therefore:

$$
2(m-1) \nabla J d \tau=\left(\lambda-\tau \lambda_{\tau}\right) \omega-\tau f d \tau \wedge d^{c} \tau
$$

and clearly $d\left(\lambda-\tau \lambda_{\tau}\right) \wedge d \tau=0$.

q.e.d.

Remark 1. More generally, if $2 \nabla J d \tau=\xi \rho+\eta \omega$ for some functions $\xi(\tau)$ and $\eta(\tau)$, then the conclusions of this lemma hold on the open set where $d \xi=\xi_{\tau} d \tau \neq 0$, with essentially the same proof [11].

In order to interpret the work of Derdziński and Maschler in the present work, we reformulate equation (10). We first note that if $\tau$ is any function satisfying (10), for some functions $p, q$ with $d p \wedge d \tau=0$, then in fact we have

$$
d\left(|d \tau|^{2}\right) \wedge d \tau=0, \quad d q \wedge d \tau=0, \quad \text { and } \quad p=\frac{a}{a \tau+b}|d \tau|^{2}
$$

for some constants $a$ and $b$ not both zero. Indeed, contracting (10) with $J d \tau$ we obtain $d\left(|d \tau|^{2}\right)=\left(p+q|d \tau|^{2}\right) d \tau$ which gives the first two observations. Hence $d d^{c} \tau=f|d \tau|^{2} \omega+q d \tau \wedge d^{c} \tau$, where $d f \wedge d \tau=0$. The exterior derivative of this equation gives $|d \tau|^{2}\left(d f+f^{2} d \tau\right) \wedge \omega=0$, so that $f=a /(a \tau+b)$.

Lemma 2. A Killing potential $\tau$ satisfies the equation

$$
d d^{c} \tau=\frac{a}{a \tau+b}|d \tau|^{2} \omega+q d \tau \wedge d^{c} \tau
$$

(for some function $q$ and constants $a, b$ not both zero) if and only if the 2 -form $\phi:=(a \tau+b) d \tau \wedge d^{c} \tau /|d \tau|^{2}$ satisfies

$$
\nabla_{X} \phi=\frac{a}{2}\left(d \tau \wedge J X-d^{c} \tau \wedge X\right) .
$$


Proof. If $\phi=(a \tau+b) d \tau \wedge d^{c} \tau /|d \tau|^{2}$, then

$$
\begin{aligned}
\nabla_{X} \phi=a d \tau(X) \frac{d \tau \wedge d^{c} \tau}{|d \tau|^{2}}- & (a \tau+b)\left\langle\iota_{X} d d^{c} \tau, d^{c} \tau\right\rangle \frac{d \tau \wedge d^{c} \tau}{|d \tau|^{4}} \\
& +(a \tau+b) \frac{\iota_{J X} d d^{c} \tau \wedge d^{c} \tau+d \tau \wedge \iota_{X} d d^{c} \tau}{2|d \tau|^{2}}
\end{aligned}
$$

This can only equal $\frac{a}{2}\left(d \tau \wedge J X-d^{c} \tau \wedge X\right)$ if $d d^{c} \tau$ is of the form $f|d \tau|^{2} \omega+$ $q d \tau \wedge d^{c} \tau$ for some functions $f, q$, in which case we obtain

$\nabla_{X} \phi=(a-(a \tau+b) f) d \tau(X) \frac{d \tau \wedge d^{c} \tau}{|d \tau|^{2}}+\frac{1}{2}(a \tau+b) f\left(d \tau \wedge J X-d^{c} \tau \wedge X\right)$.

The result is now immediate.

q.e.d.

\section{Hamiltonian 2-forms}

In this section we introduce the notion of a hamiltonian 2-form and develop the most basic general properties and the simplest examples.

2.1. Hamiltonian 2-forms and Killing vector fields. The definition of hamiltonian 2-forms is motivated both by weakly Bochner-flat Kähler manifolds and by strongly conformally Einstein Kähler manifolds. The reason for the terminology will shortly become apparent.

Definition 2. Let $\phi$ be any (real) $J$-invariant 2-form on the Kähler manifold $(M, g, J, \omega)$. We say $\phi$ is hamiltonian if there is a function $\sigma$ on $M$ such that

$$
\nabla_{X} \phi=\frac{1}{2}\left(d \sigma \wedge J X-d^{c} \sigma \wedge X\right)
$$

for any vector field $X$. When $M$ is a Riemann surface, we require in addition that $\sigma$ is a Killing potential.

It follows immediately from the definition that $d \sigma=d \operatorname{tr} \phi$, where $\operatorname{tr} \phi=\langle\phi, \omega\rangle$ is the trace of $\phi$ with respect to $\omega$, so without loss of generality we may take $\sigma=\operatorname{tr} \phi$. The defining equation for hamiltonian 2 -forms is therefore linear. Note that, for a general hamiltonian 2-form $\phi, A=\phi+\sigma \omega$ is closed, $d A=0$.

Example 1. On any Kähler manifold, any $J$-invariant parallel 2form is hamiltonian. In particular, a constant multiple of the Kähler form $\omega$ is hamiltonian. It follows that if $\phi$ is hamiltonian, then so is $\phi_{t}:=\phi-t \omega$ for any constant $t$.

Example 2. We shall be particularly interested in the hamiltonian 2forms arising from the following immediate consequence of equation (8).

Proposition 1. A Kähler manifold of dimension $2 m \geq 4$ is weakly Bochner-flat if and only if the normalized Ricci form $\tilde{\rho}=\rho_{0}+\frac{1}{m} s \omega=$ $\rho-s \omega$ is hamiltonian. 
Example 3. In view of equation (11), we also have the following result.

Proposition 2. On any strongly conformally Einstein Kähler manifold of dimension $2 m \geq 4$ with conformal factor $\tau$, there are constants $a, b$ not both zero such that $\phi=(a \tau+b) d \tau \wedge J d \tau /|d \tau|^{2}$ is hamiltonian on the open set where $d \tau$ is nonzero.

The equation for hamiltonian 2-forms is overdetermined. By differentiating and skew-symmetrizing (12), we get

$$
\begin{aligned}
R_{X, Y} \cdot \phi=\left[R_{X, Y}, \phi\right]= & \frac{1}{2}\left(\nabla_{Y} d \sigma \wedge J X-\nabla_{X} d \sigma \wedge J Y\right. \\
& \left.-J \nabla_{Y} d \sigma \wedge X+J \nabla_{X} d \sigma \wedge Y\right) .
\end{aligned}
$$

This formula underlies most of the basic theory of hamiltonian 2-forms. In particular, we shall use it to explain the use of the term "hamiltonian" in this context.

To do that, we first recall that the pfaffian of a 2 -form $\phi$ is defined by

$$
\text { pf } \phi=\frac{1}{m !} *(\phi \wedge \cdots \wedge \phi),
$$

where $*$ denotes the Hodge star operator. The normalization is chosen so that $\operatorname{pf} \omega=1$ and thus $\phi \wedge \cdots \wedge \phi=(\operatorname{pf} \phi) \omega \wedge \cdots \wedge \omega$. We let $\phi_{t}=\phi-t \omega$ as in Example 1 above, and (following Bryant [8]) define the momentum polynomial of $\phi$ to be

$$
p(t):=(-1)^{m} \operatorname{pf} \phi_{t}=t^{m}-(\operatorname{tr} \phi) t^{m-1}+\cdots+(-1)^{m} \operatorname{pf} \phi .
$$

Proposition 3. If $\phi$ is a hamiltonian 2-form, then the functions $p(t)$ on $M$ (for each $t \in \mathbb{R})$ are Poisson-commuting hamiltonians for Killing vector fields $K(t):=J \operatorname{grad}_{g} p(t)$ which preserve $\phi$. In particular, the vector fields $K(t)$ all commute.

Proof. We first prove that $K:=J \operatorname{grad}_{g} \sigma$ is Killing, i.e., $\nabla d \sigma$ is $J$ invariant. Since $R_{X, Y}$ is $J$-invariant in $X$ and $Y$, equation (13) implies that

$$
\begin{array}{cc}
\text { (16) } & S(X) \wedge J Y-J S(X) \wedge Y-S(Y) \wedge J X+J S(Y) \wedge X=0, \\
\text { where } & S(X)=\nabla_{X} d \sigma+J \nabla_{J X} d \sigma .
\end{array}
$$

Contracting (16) with a vector field $Z$ and taking the trace over $Y$ and $Z$ yields $2(1-m) J S(X)=0$ and hence $\nabla d \sigma$ is $J$-invariant-by definition when $m=1$.

We now show that the other hamiltonian vector fields are Killing. To do this we differentiate pf $\phi_{t}$, using the fact that $\phi_{t}$ is hamiltonian with $\operatorname{tr} \phi_{t}=\operatorname{tr} \phi-m t$ and hence $d \operatorname{tr} \phi_{t}=d \sigma$. Therefore, from (12) and (14), 
we get

$$
d \operatorname{pf} \phi_{t}=\frac{1}{(m-1) !} *\left(J d \sigma \wedge \phi_{t} \wedge \cdots \wedge \phi_{t}\right)
$$

Using (12) again, we then obtain

$$
\begin{aligned}
\nabla_{X} d \operatorname{pf} \phi_{t}= & \frac{1}{(m-1) !} *\left(\nabla_{X} J d \sigma \wedge \phi_{t} \wedge \cdots \wedge \phi_{t}\right) \\
& +\frac{1}{2(m-2) !} *\left(J X \wedge d \sigma \wedge J d \sigma \wedge \phi_{t} \wedge \cdots \wedge \phi_{t}\right) .
\end{aligned}
$$

The second term on the right hand side is automatically $J$-invariant, while the first one is also $J$-invariant since $K$ is Killing. Hence $J \operatorname{grad}_{g} p(t)$ is Killing for all $t$.

It remains to prove that the Killing vector fields preserve $\phi$ and that their momentum maps Poisson-commute. Contracting equation (17) with $J d \sigma$, we deduce that $\left\langle J d \operatorname{pf} \phi_{t}, d \sigma\right\rangle=0$ and hence $K(t)$ preserves $\sigma$ for all $t$. It follows that $\mathcal{L}_{K(t)} \phi=\mathcal{L}_{K(t)}\left(\phi_{t}+\sigma \omega\right)=d \iota_{K(t)}\left(\phi_{t}+\sigma \omega\right)$, since $\phi_{t}+\sigma \omega$ is closed.

Now equation (17) also implies that

$$
\phi_{t}\left(\operatorname{grad}_{g}\left(\operatorname{pf} \phi_{t}\right), \cdot\right)=\frac{1}{m !} \sum_{j=1}^{2 m}\left\langle J d \sigma, * \iota_{e_{j}}\left(\phi_{t} \wedge \cdots \wedge \phi_{t}\right)\right\rangle \varepsilon_{j}=\left(\operatorname{pf} \phi_{t}\right) J d \sigma
$$

(using a local frame $e_{j}$ with dual frame $\varepsilon_{j}$ ) so that

$$
\phi_{t}\left(J \operatorname{grad}_{g} p(t), \cdot\right)=-p(t) d \sigma .
$$

Hence $\iota_{K(t)}\left(\phi_{t}+\sigma \omega\right)=-d(\sigma p(t))$ is closed and so $K(t)$ preserves $\phi$. It follows that $K(t)$ preserves $p(s)$ for all $s, t \in \mathbb{R}$ and $\{p(s), p(t)\}=$ $\langle J K(s), K(t)\rangle=d(p(s))(K(t))=0$ for all $s, t$; thus $p(s)$ and $p(t)$ Poisson-commute.

q.e.d.

Obviously $p(t)$ is a Killing potential for all $t$ if and only if its coefficients are all Killing potentials.

2.2. The connection for 2-jets of hamiltonian 2-forms. We have noted already that the equation for hamiltonian 2-forms is overdetermined. In fact it has finite type, i.e., the space of local solutions is finite dimensional, the 2-jets of hamiltonian 2-forms being the parallel sections with respect to a certain connection.

Proposition 4. If $\phi$ is a hamiltonian 2-form then

$$
\begin{gathered}
\nabla \phi+\frac{1}{2}(K \wedge \mathrm{Id}+J K \wedge J)=0 \\
\nabla K+\frac{1}{2 m}(2 u \omega-J\{\rho, \phi\}-2 R(\phi))=0 \\
d u+\rho(K)=0 .
\end{gathered}
$$


(Here, as elsewhere, we identify vectors with 1-forms and bilinear forms with endomorphisms using $g$, and we recall that $\{\cdot, \cdot\}$ denotes the anticommutator.)

Thus $(\phi, K, u)$-with $K=J \operatorname{grad}_{g} \sigma, u=\frac{1}{2} \Delta \sigma$ and $\sigma=\operatorname{tr} \phi-i s$ parallel with respect to a natural covariant derivative $\mathcal{D}$ on $\Lambda^{J,+} M \oplus$ $T M \oplus M \times \mathbb{R}$.

The integrability condition $F^{\mathcal{D}} \cdot(\phi, K, u)=0$ is equivalent to the equations

$$
\begin{aligned}
m[R(\psi), \phi]-\left[R(\phi)+\frac{1}{2} J\{\rho, \phi\}, \psi^{J,+}\right] & =0 \\
(m+1) R_{K, X}-\rho(K, X) \omega+\frac{1}{2} J\left\{\rho, K \wedge X^{J,+}\right\} & \\
-\frac{1}{2} J\left\{\nabla_{X} \rho, \phi\right\}-\nabla_{X} R(\phi) & =0 \\
-m \nabla_{K} \rho+[R(\phi), \rho] & =0 .
\end{aligned}
$$

for any 2 -form $\psi$ and vector field $X$. Note that (23) with $\psi=\omega$ gives $[\rho, \phi]=0$.

Proof. Equation (20) is immediate by definition. Contracting (13) with a vector field $Z$ and taking the trace over $Y$ and $Z$ gives $\sum_{j=1}^{2 m} R_{X, e_{j}} \phi\left(e_{j}\right)+\phi(\operatorname{Ric}(X))=-\frac{1}{2}(\Delta \sigma) J X-m J \nabla_{X} d \sigma$ (for a local orthonormal frame $e_{j}$ ) and (21) is the $J$-invariant part of this (as $\nabla d \sigma$ is $J$-invariant). Since $K$ is a Killing vector field, $\nabla_{X} \nabla K=R_{K, X}[\mathbf{1 8}]$, and $(22)$ is obtained by contracting this with $\omega$.

The first integrability condition (23) follows from (13) by substituting for $\nabla K=J \nabla d \sigma$. Differentiating (21) using $\nabla_{X} \nabla K=R_{K, X}$ and the equations for $\nabla_{X} \phi$ and $d u(X)$ gives (24). Finally, from equation (22), $0=d(\rho(K))=\mathcal{L}_{K} \rho=\nabla_{K} \rho-[\nabla K, \rho]$, which yields $(25)$ by substituting for $\nabla K$.

The three components of $F^{\mathcal{D}} \cdot(\phi, K, u)$ are the left hand sides of $(23)$ (25) divided by $m$, after applying the isomorphism alt: $T^{*} M \otimes \Lambda^{2} M \rightarrow$ $\Lambda^{2} M \otimes T M$ to $(24)$.

q.e.d.

Remark 2. It follows that hamiltonian 2-forms enjoy the properties of parallel sections, such as unique continuation, extendibility to submanifolds of codimension at least two, and an upper bound, here equal to $m^{2}+2 m+1$, on the dimension of space of hamiltonian 2 -forms.

We now expand the curvature $R$ using (3), which may be rewritten

$$
R(\psi)=W^{\mathcal{K}}(\psi)-J\left\{\hat{\rho}, \psi^{J,+}\right\}+\langle\hat{\rho}, \psi\rangle \omega+(\operatorname{tr} \psi) \hat{\rho}
$$

for any 2 -form $\psi$, where $\hat{\rho}=\frac{1}{m+2}\left(\tilde{\rho}+\frac{1}{2} s \omega\right)=\frac{1}{m+2}\left(\rho-\frac{1}{2} s \omega\right)$. Then (21) reads

$$
\begin{aligned}
& \nabla K=\frac{1}{2} J\{\hat{\rho}, \phi\} \\
& \quad+\frac{1}{m}\left(W^{\mathcal{K}}(\phi)+(\operatorname{tr} \phi) \hat{\rho}-(\operatorname{tr} \hat{\rho}) \phi+(\langle\hat{\rho}, \phi\rangle-u) \omega\right) .
\end{aligned}
$$


Using $[\rho, \phi]=0$, equation (23) implies

$$
\left[W^{\mathcal{K}}(\psi), \phi\right]=\frac{1}{m}\left[W^{\mathcal{K}}(\phi), \psi^{J,+}\right]+J\left(\hat{\rho}_{0} \circ \psi^{J,+} \circ \phi_{0}-\phi_{0} \circ \psi^{J,+} \circ \hat{\rho}_{0}\right)
$$

$\left(\hat{\rho}_{0}=\frac{\rho_{0}}{m+2}\right)$. Equation (24) is complicated when fully expanded. Instead we use the fact that the $J$-invariant part of $K \wedge X$ is $-\nabla_{X} \phi$ to obtain

$$
R_{K, X}=W_{K, X}^{\mathcal{K}}+J\left\{\hat{\rho}, \nabla_{X} \phi\right\}-\left\langle\hat{\rho}, \nabla_{X} \phi\right\rangle \omega-d \sigma(X) \hat{\rho}
$$

and $d u(X)=-\rho(K, X)=(m+2)\left\langle\hat{\rho}, \nabla_{X} \phi\right\rangle+d \sigma(X) \operatorname{tr} \hat{\rho}$. Substituting these into the covariant derivative of (27) (using $\nabla_{X} \nabla K=R_{K, X}$ as before) we have

$$
\begin{aligned}
& W_{K, X}^{\mathcal{K}}-\frac{1}{m} \nabla_{X}\left(W^{\mathcal{K}}(\phi)\right) \\
& =\frac{1}{2} J\left\{\nabla_{X} \hat{\rho}_{0}, \phi_{0}\right\}+\frac{1}{m}\left\langle\nabla_{X} \hat{\rho}_{0}, \phi_{0}\right\rangle \omega-\frac{1}{m} d s(X) \phi_{0} \\
& \quad-\frac{1}{2} J\left\{\hat{\rho}_{0}, \nabla_{X} \phi_{0}\right\}-\frac{1}{m}\left\langle\hat{\rho}_{0}, \nabla_{X} \phi_{0}\right\rangle \omega+\frac{1}{m} d \sigma(X) \rho_{0} .
\end{aligned}
$$

The important point we shall need later is that the right hand side vanishes if $\tilde{\rho}$ is a constant linear combination of $\phi$ and $\omega$.

2.3. The differential system in the weakly Bochner-flat case. On a weakly Bochner-flat Kähler manifold, the normalized Ricci form $\tilde{\rho}$ is hamiltonian. We also want to study hamiltonian 2-forms on KählerEinstein manifolds. These cases can be considered together by supposing that $(g, J, \omega)$ is a weakly Bochner-flat Kähler metric with a hamiltonian 2 -form $\phi$ such that $\tilde{\rho}$ is a constant linear combination of $\phi$ and $\omega$. We set $\tilde{\rho}=(m+2) a \phi+b \omega$, and find that (27) may be written

$\nabla K=\frac{1}{m} W^{\mathcal{K}}(\phi)+a J \circ \phi^{2}-(a \sigma+b) \phi+\frac{1}{m}\left(a\left(\sigma^{2}+\langle\phi, \phi\rangle\right)+b \sigma-\frac{1}{2} \Delta \sigma\right) \omega$.

Let us put $\tau_{0}=-2 a, \tau_{1}=-2(a \sigma+b)$ and $\tau_{2}=\frac{2}{m}\left(a\left(\sigma^{2}+\langle\phi, \phi\rangle\right)+b \sigma-\right.$ $\left.\frac{1}{2} \Delta \sigma\right)$. Then, as $d\langle\phi, \phi\rangle=-2 \phi(K)$, we obtain the following formulation of the system (20)-(22):

$$
\begin{aligned}
\nabla \phi & =-\frac{1}{2}(K \wedge \operatorname{Id}+J K \wedge J) \\
\nabla K & =\frac{1}{m} W^{\mathcal{K}}(\phi),-\frac{1}{2} \tau_{0} J \circ \phi^{2}+\frac{1}{2} \tau_{1} \phi+\frac{1}{2} \tau_{2} \omega, \\
d \tau_{2} & =-\tau_{0} \phi(K)-\tau_{1} J K, \quad d \tau_{1}=-\tau_{0} J K, \quad d \tau_{0}=0 .
\end{aligned}
$$

When $W^{\mathcal{K}}(\phi)=0$ this system yields an invariant polynomial-in particular for $\tau_{0} \neq 0$ and $W^{\mathcal{K}}=0$, such a polynomial was found by Bryant [8] and is the basis for his classification of Bochner-flat Kähler metrics.

Proposition 5. Let $\left(\phi, K, \tau_{0}, \tau_{1}, \tau_{2}\right)$ be a solution of $(30)$ with $W^{\mathcal{K}}(\phi)$ $=0$ and define a polynomial

$$
F_{\mathrm{c}}(t):=\left(\tau_{0} t^{2}+\tau_{1} t+\tau_{2}\right) p(t)-\langle K, K(t)\rangle .
$$


Then $F_{\mathrm{c}}(t)$ has constant coefficients. (Recall that $K(t)=J \operatorname{grad}_{g} p(t)$, where $p(t)=(-1)^{m} \operatorname{pf} \phi_{t}$ and $\phi_{t}=\phi-t \omega$.)

Proof. Equation (17) implies that $\langle K, J X\rangle p(t)=\left\langle\phi_{t}(X), K(t)\right\rangle$. Hence differentiating $\left(\tau_{0} t^{2}+\tau_{1} t+\tau_{2}\right) p(t)$ along a vector field $X$, using the system (30), gives

$$
\begin{aligned}
\langle K & \left., \tau_{0} t J X\right\rangle p(t)+\left\langle K, \tau_{0} \phi(X)+\tau_{1} J X\right\rangle p(t) \\
& +\left(\tau_{0} t^{2}+\tau_{1} t+\tau_{2}\right)\langle J X, K(t)\rangle \\
= & \left\langle\left(\tau_{0} t \phi_{t}-\tau_{0} \phi_{t} \circ \phi \circ J+\tau_{1} \phi_{t}+\tau_{0} t^{2} J+\tau_{1} t J+\tau_{2} J\right)(X), K(t)\right\rangle \\
= & \left\langle\left(-\tau_{0} J \circ \phi^{2}+\tau_{1} \phi+\tau_{2} J\right)(X), K(t)\right\rangle=2\left\langle\nabla_{X} K, K(t)\right\rangle .
\end{aligned}
$$

Now (18) gives $\left\langle K, \nabla_{X}(K(t))\right\rangle=\left\langle\nabla_{X} K, K(t)\right\rangle$, which proves the proposition.

q.e.d.

Following Bryant, we refer to $F_{\mathrm{c}}$ as the characteristic polynomial of $(g, J, \omega, \phi)$.

2.4. Complex projective, hyperbolic and euclidean space. A Kähler metric $g$ has constant holomorphic sectional curvature if and only if it is Bochner-flat and Kähler-Einstein (and we require constant scalar curvature when $m=1)$. It follows from (23)-(25) that the connection $\mathcal{D}$ is flat in this case; hence on any simply connected domain, the space of hamiltonian 2-forms has dimension $(m+1)^{2}$. Conversely, if $\mathcal{D}$ is flat, then $\rho_{0}=0$ (as $\left[\rho_{0}, \phi\right]=0$ for all $\left.\phi \in \Lambda^{J,+} M\right)$; now (28) implies that $W^{\mathcal{K}}=0$ (since $\left[W^{\mathcal{K}}(\phi), \psi\right]=0$ for all $\left.\phi, \psi \in \Lambda^{J,+} M\right)$; finally $(29)$ gives $d s=0$ (even if $m=1$ ), so $g$ has constant holomorphic sectional curvature.

Hamiltonian 2-forms on constant holomorphic sectional curvature manifolds correspond to solutions of (30) with $W^{\mathcal{K}}=0, \tau_{0}=0$ and $\tau_{1}=-2 s / m$. We first consider the case that $s$ is nonzero, i.e., up to scale, the Kähler metric is the Fubini-Study metric of complex projective space, or the Bergman metric of complex hyperbolic space. If we put $\tau_{2}=2 s \tau / m$, the system (30) becomes

$$
\begin{aligned}
\nabla \phi & =-\frac{1}{2}(K \wedge \mathrm{Id}+J K \wedge J) \\
\nabla K & =-\frac{s}{m}(\phi-\tau \omega) \\
d \tau & =J K .
\end{aligned}
$$

The last two equations show that $\tau$ is a Killing potential for $-K$, and that the hamiltonian 2-form $\phi$ is completely determined by $\tau$. Furthermore, the Kostant identity $\nabla_{X} \nabla K=R_{K, X}$ shows that any Killing potential defines a hamiltonian 2-form in this way. Hence there is a bijection $\phi \mapsto \frac{1}{m} \sigma-\frac{1}{2 s} \Delta \sigma$ (with inverse $\tau \mapsto \frac{m}{2 s} d d^{c} \tau+\tau \omega$ ) from the space hamiltonian 2-forms to the space of Killing potentials, which may 
be identified with the unitary Lie algebra $\mathfrak{u}(m+1)$ or $\mathfrak{u}(m, 1)$, using the Poisson bracket. We remark, though we shall not make use of this, that the Lie bracket, Killing form and (monic, degree $m+1$ ) characteristic polynomial can be computed and turn out to be given by

$$
\begin{aligned}
{[(\phi, K, \tau),(\hat{\phi}, \hat{K}, \hat{\tau})]=} & \left([\phi, \hat{\phi}]+\frac{m}{s} K \wedge \hat{K}^{J,+},\right. \\
& \left.\iota_{\hat{K}}(\phi-\tau \omega)-\iota_{K}(\hat{\phi}-\hat{\tau} \omega), \frac{m}{s} \omega(K, \hat{K})\right) \\
\langle(\phi, K, \tau),(\hat{\phi}, \hat{K}, \hat{\tau})\rangle= & \langle\phi, \hat{\phi}\rangle+\tau \hat{\tau}+\frac{m}{s}\langle K, \hat{K}\rangle \\
b P_{(\phi, K, \tau)}(t)= & -\frac{m}{2 s} F_{\mathrm{c}}(t)=(t-\tau) p(t)+\frac{m}{2 s}\langle K, K(t)\rangle .
\end{aligned}
$$

On a flat Kähler manifold (e.g., on complex euclidean space $\mathbb{C}^{m}$ ) the system (30) reduces to

$$
\begin{aligned}
\nabla \phi & =-\frac{1}{2}(K \wedge \mathrm{Id}+J K \wedge J) \\
\nabla K & =\frac{1}{2} \kappa \omega,
\end{aligned}
$$

with $\kappa$ constant. Thus inside the space of hamiltonian 2 -forms we have the parallel 2-forms $(K=0)$; modulo such parallel 2-forms, we then have the parallel vector fields $(\kappa=0)$; then finally, the space of hamiltonian 2 -forms on $\mathbb{C}^{m}$, modulo those with $K$ parallel, is one dimensional, a representative element being $d t \wedge d^{c} t$, where $t$ is the distance squared to the origin. The characteristic polynomial is now

$$
F_{\mathrm{c}}(t)=\kappa p(t)+\frac{1}{2}\langle K, K(t)\rangle
$$

which has degree $m$ if $\kappa \neq 0$, degree $m-1$ if $\kappa=0$ and $K \neq 0$, and is zero if $K=0$.

We shall obtain an explicit description of the hamiltonian 2-forms on complex projective, hyperbolic and euclidean space, with a given characteristic polynomial $F_{\mathrm{c}}(t)$, in section 5.4 below.

\section{Hamiltonian torus actions}

We have seen that on a Kähler 2m-manifold with a hamiltonian 2form, there is a family of Poisson-commuting hamiltonian Killing vector fields $K(t)=J \operatorname{grad}_{g} p(t)$. Since $p(t)$ is a monic polynomial of degree $m$, the span of the $K(t)$ is at most $m$-dimensional. If they are not all zero, then on an open set where the span has rank $\ell, 1 \leq \ell \leq m$, the $K(t)$ generate a local action of an $\ell$-dimensional torus.

We next study hamiltonian $\ell$-torus actions in general. Our discussion is independent of the theory of hamiltonian 2-forms, but is strongly motivated by it. Roughly speaking, there are three aspects to the description of such torus actions: first, the toric geometry of the fibres of the complexified action; second, the geometry of the base of this action, the local Kähler quotient; third, the way the fibre and base geometries 
fit together. In full generality, these structures are quite difficult to handle. However, there is a class of toric manifolds, called orthotoric, of Kähler quotients, called semisimple, and of fibrations, called rigid, which are more amenable to computation. It will turn out that the hamiltonian torus actions induced by hamiltonian 2-forms are always rigid with semisimple base and orthotoric fibres.

\subsection{The Pedersen-Poon construction.}

Definition 3. A local (isometric) hamiltonian $\ell$-torus action on a Kähler $2 m$-manifold $(M, g, J, \omega)$ is an $\ell$-dimensional family of holomorphic Killing vector fields $\boldsymbol{K} \in C^{\infty}(M, T M) \otimes \mathbb{R}^{\ell *}$ which are linearly independent on a dense open set $M^{0}$ and isotropic in the sense that $\omega(\boldsymbol{K}, \boldsymbol{K})=0$. The last condition means that every component of $J \boldsymbol{K}$ is orthogonal to every component of $\boldsymbol{K}$. It follows that $\ell \leq m$-if equality holds, we say that $(M, g, J, \omega)$ is a toric Kähler manifold.

For clarity, we write $K_{r}=\boldsymbol{K}\left(e_{r}\right)(r=1, \ldots \ell)$ for the components of $\boldsymbol{K}$ with respect to a basis $e_{r}$ of $\mathbb{R}^{\ell}$ - this could also be interpreted as an abstract index notation. In this subsection and the next two (only) we adopt the summation convention, i.e., repeated indices imply contraction.

Since $\mathcal{L}_{K_{r}} \omega=0$ for all $r$ and $\omega$ is closed, we have $d\left(\iota_{K_{r}} \omega\right)=0$ and $\iota_{\left[K_{r}, K_{s}\right]} \omega=-d\left(\omega\left(K_{r}, K_{s}\right)\right)=0$. Furthermore, since $\mathcal{L}_{K_{r}} J=0$ for all $r$ and $J$ is integrable, we have $\mathcal{L}_{J K_{r}} J=0$ and $\left[J K_{r}, J K_{s}\right]=J\left[J K_{r}, K_{s}\right]=$ 0 .

Remark 3. Definition 3 can be extended to almost hermitian manifolds, but if $\omega$ is not closed, we assume a priori that $\left[K_{r}, K_{s}\right]=0$ for all $r, s$, while if $J$ is not integrable, we assume that $\left[J K_{r}, J K_{s}\right]$ is in the span of $J \boldsymbol{K}$ for all $r, s$.

To obtain a local description of these metrics, valid near any point in $M^{0}$, we may assume that $\boldsymbol{K}$ generates a free $\ell$-torus action, so that $M$ is a principal $\ell$-torus bundle over a $(2 m-\ell)$-dimensional manifold $B$, and that the foliation generated by $\boldsymbol{K}, J \boldsymbol{K}$ descends to a fibration of $B$ over a $2(m-\ell)$-dimensional manifold $S$.

Since $J$ is integrable and $\boldsymbol{K}$-invariant, the components of $J \boldsymbol{K}$ are holomorphic vector fields, so that $S$ is a complex manifold.

Further, since $\omega$ is closed and $\boldsymbol{K}$-invariant, we may locally write $\iota_{\boldsymbol{K}} \omega=-d \boldsymbol{\sigma}$ where $\boldsymbol{\sigma}: M \rightarrow \mathbb{R}^{\ell *}$ is a $\boldsymbol{K}$-invariant momentum map for the torus action. Thus we may locally identify $B$ with $S \times U$, where $U$ is an open subset of $\mathbb{R}^{\ell *}$ and $\boldsymbol{\sigma}$ is given by projection to $U$. $S$ is then the Kähler quotient of $M$ : it is a complex manifold equipped with a family of compatible Kähler structures parameterized by $U$.

It is useful to split the exterior derivative on $B$ into horizontal and vertical parts:

$$
d \alpha=d_{h} \alpha+d \sigma_{r} \wedge \mathcal{L}_{\partial / \partial \sigma_{r}} \alpha
$$


(Note $\mathcal{L}_{\partial / \partial \sigma_{r}}$ commutes with $d_{h}$. We write $\partial \alpha / \partial \sigma_{r}$ as a shorthand for $\mathcal{L}_{\partial / \partial \sigma_{r}} \alpha$.)

Let $\boldsymbol{\theta} \in C^{\infty}\left(M, \Lambda^{1} M\right) \otimes \mathbb{R}^{\ell}$ be the connection 1-forms dual to $\boldsymbol{K}$ and let $J \boldsymbol{\theta}=-\boldsymbol{\theta} \circ J$; thus $\theta_{r}\left(K_{s}\right)=\delta_{r s}$ and $\theta_{r}$ vanishes on the horizontal distribution of $M \rightarrow B$. (We may locally write $\theta_{r}=d t_{r}+\alpha_{r}$ where $\boldsymbol{t}: M \rightarrow \mathbb{R}^{\ell}$ and $\left.\alpha_{r}\left(K_{s}\right)=0=\alpha_{r}\left(J K_{s}\right).\right)$

The two families of 1 -forms $J \boldsymbol{\theta}$ and $\iota_{\boldsymbol{K}} \boldsymbol{\omega}$ span the same $\ell$-dimensional space. Hence we may write

$$
J \theta_{r}=G_{r s} \iota_{K_{s}} \omega \quad \text { and } \quad \iota_{K_{r}} \omega=H_{r s} J \theta_{s},
$$

where $G_{r s}$ and $H_{r s}$ are mutually inverse and $\boldsymbol{K}$-invariant. Since $\left\langle K_{r}, K_{s}\right\rangle$ $=\omega\left(K_{r}, J K_{s}\right)=H_{r s}$, we deduce that $G_{r s}$ and $H_{r s}$ are symmetric and positive definite.

Proposition $6([\mathbf{2 2}])$. Let $(S, J)$ be a complex $2(m-\ell)$-manifold, let $B=S \times U$ with $U$ open in $\mathbb{R}^{\ell *}$, and let $M$ be a principal $\ell$-torus bundle over $B$. Denote the components of the projection $\boldsymbol{\sigma}: B \rightarrow \mathbb{R}^{\ell *}$ by $\sigma_{r}$. Now suppose that:

(i) $\left(h, \omega_{h}\right)$ is family of compatible Kähler metrics on the level surfaces of $\boldsymbol{\sigma}$ in $B$;

(ii) $G_{r s}$ is a symmetric positive definite matrix of functions on $B$, with inverse matrix $H_{r s}$, satisfying the equations

$$
\frac{\partial G_{r s}}{\partial \sigma_{t}}=\frac{\partial G_{r t}}{\partial \sigma_{s}} \quad \text { and } \quad d_{h} d_{h}^{c} G_{r s}+\frac{\partial^{2} \omega_{h}}{\partial \sigma_{r} \partial \sigma_{s}}=0 ;
$$

(iii) $\boldsymbol{\theta}: M \rightarrow \mathbb{R}^{\ell}$ is the connection 1-form of a principal connection on $M$ over $B$ whose curvature satisfies the equation

$$
d \theta_{r}=\frac{\partial \omega_{h}}{\partial \sigma_{r}}+d_{h}^{c} G_{r s} \wedge d \sigma_{s} .
$$

Then the almost hermitian structure

$$
\begin{aligned}
g & =h+G_{r s} d \sigma_{r} d \sigma_{s}+H_{r s} \theta_{r} \theta_{s} \\
\omega & =\omega_{h}+d \sigma_{r} \wedge \theta_{r} \\
J \theta_{r} & =-G_{r s} d \sigma_{s}
\end{aligned}
$$

on $M$ is Kähler with a free hamiltonian $\ell$-torus action and Kähler quotient $S$.

Any Kähler manifold with a local hamiltonian $\ell$-torus action arises locally in this way on the dense open set $M^{0}$ where the Killing vector fields are independent.

Proof. We have seen already that any Kähler structure with a local hamiltonian $\ell$-torus action can be written in the form (36), where $\left(h, J, \omega_{h}\right)$ is Kähler for each fixed $\boldsymbol{\sigma}$ and $d \theta_{r}\left(J K_{s}, J K_{t}\right)=$ $-\theta_{r}\left(\left[J K_{s}, J K_{t}\right]\right)=0$. Now under these conditions, $\zeta_{r}=G_{r s} d \sigma_{s}+i \theta_{r}$ generate the $(1,0)$-forms on the fibres, so $(g, J, \omega)$ is Kähler if and only 
if the $\zeta_{r}$ generate a differential ideal modulo horizontal forms and $\omega$ is closed. Since $d \omega=\left(\partial \omega_{h} / \partial \sigma_{r}-d \theta_{r}\right) \wedge d \sigma_{r}$, and $d \zeta_{r}=d_{h} G_{r s} \wedge d \sigma_{s}-$ $\left(\partial G_{r s} / \partial \sigma_{t}\right) d \sigma_{s} \wedge d \sigma_{t}+i d \theta_{r}$, it follows easily that $(g, J, \omega)$ is Kähler if and only if $G_{r s}$ satisfies (34) and $\theta_{r}$ satisfies (35): the second part of (34) follows from the integrability of (35).

q.e.d.

Remark 4. It is crucial here that the local torus action is hamiltonian in the strong sense that the components of $\boldsymbol{\sigma}$ Poisson-commute, i.e., $d \sigma_{r}\left(K_{s}\right)=0$. This condition is often missed in the literature, since if $K_{r}$ and $K_{s}$ commute, $d \sigma_{r}\left(K_{s}\right)$ is constant, and on a compact manifold $\sigma_{r}$ must have a critical point, so the constant is zero. However, we are not assuming compactness: indeed, the above local description is only valid on an open set where $\sigma_{r}$ has no critical points!

Proposition 6 shows that a Kähler metric with a local hamiltonian $\ell$-torus action may be specified by essentially free data. Indeed (35) is integrable by virtue of (34). To solve the latter, observe that the first part implies we can write $G_{r s}=\partial u_{r} / \partial \sigma_{s}$, and since $G_{r s}$ is symmetric, $u_{r}=\partial G / \partial \sigma_{r}$ for some function $G$ on $B$ such that the $d_{h}$-closed $J$ invariant 2-form $\omega_{h}+d_{h} d_{h}^{c} G$ depends affinely on $\boldsymbol{\sigma}$. Since we can add a $d_{h} d_{h}^{c}$ potential for this 2 -form to $G$ without altering $G_{r s}$, we can assume (locally) that for each fixed $\boldsymbol{\sigma}, \omega_{h}+d_{h} d_{h}^{c} G=0$. Thus $G$ determines $\omega_{h}$ and $G_{r s}$ and is now subject only to the open condition that these are positive definite.

In fact $G$ is a fibrewise Legendre transform of a Kähler potential, generalizing work of Guillemin in the toric case [14] (see also [1]). Observe first that $d^{c} u_{r}=d_{h}^{c} u_{r}+\theta_{r}$, and so $d d^{c} u_{r}=\partial \omega_{h} / \partial \sigma_{r}+d_{h} d_{h}^{c} u_{r}=0$, i.e., $u_{r}$ is pluriharmonic. Now $d d^{c}\left(\sigma_{r} u_{r}-G\right)=d\left(\sigma_{r} \wedge d^{c} u_{r}-d_{h}^{c} G\right)=$ $d \sigma_{r} \wedge \theta_{r}-d_{h} d_{h}^{c} G=\omega$, so $H:=\sigma_{r} u_{r}-G$ is a Kähler potential. Since $G_{r s}$ is nondegenerate, the $u_{r}$ also form a coordinate system on each fibre of $B$ over $S$, and we let $\partial / \partial u_{r}=H_{r s} \partial / \partial \sigma_{s}$ be the coordinate vector fields tangent to the fibres, so that $H_{r s}=\partial \sigma_{s} / \partial u_{r}$ and $\sigma_{s}=\partial H / \partial u_{r}$. If we locally set $d^{c} u_{r}=d t_{r}$ then $\boldsymbol{u}+i \boldsymbol{t}: M \rightarrow \mathbb{C}^{\ell}$ is holomorphic and $\theta_{r}=d t_{r}+\alpha_{r}$ with $\alpha_{r}=-d_{h}^{c} u_{r}$. This is the fibrewise Legendre dual coordinate system to $(\boldsymbol{\sigma}, \boldsymbol{t}): M \rightarrow \mathbb{R}^{\ell *} \times \mathbb{R}^{\ell}$, and we refer to $G$ as a dual potential.

It is convenient to introduce a fixed ( $\boldsymbol{\sigma}$-independent) volume form $\operatorname{vol}_{S}$ on $S$ and write $\operatorname{vol}_{\omega_{h}}=Q \operatorname{vol}_{S}$. Observe in particular that

$$
\left\langle\omega_{h}, \frac{\partial \omega_{h}}{\partial \sigma_{r}}\right\rangle_{h}=\left\langle\omega_{h}^{-1}, \frac{\partial \omega_{h}}{\partial \sigma_{r}}\right\rangle=\frac{1}{Q} \frac{\partial Q}{\partial \sigma_{r}} .
$$

Proposition 7. Let $(M, g, J, \omega)$ be Kähler with a local hamiltonian $\ell$-torus action. 
(i) Let $f$ be any invariant function on $M$. Then

$$
\begin{aligned}
d d^{c} f= & d_{h} d_{h}^{c} f+\frac{\partial f}{\partial u_{r}} \frac{\partial \omega_{h}}{\partial \sigma_{r}} \\
& +d_{h}\left(\frac{\partial f}{\partial u_{r}}\right) \wedge \theta_{r}+d_{h}^{c}\left(\frac{\partial f}{\partial u_{r}}\right) \wedge J \theta_{r} \\
& +\frac{\partial}{\partial \sigma_{r}}\left(\frac{\partial f}{\partial u_{s}}\right) d \sigma_{r} \wedge \theta_{s}, \\
\Delta f= & \Delta_{h} f-\frac{1}{Q} \frac{\partial}{\partial \sigma_{r}}\left(Q \frac{\partial f}{\partial u_{r}}\right) .
\end{aligned}
$$

It follows that $d d^{c} f=0$ if and only if $f=A_{r} u_{r}+B$ where the $A_{r}$ are constant, and $B$ is a pluriharmonic function on $S$.

(ii) Suppose that $\kappa_{h}$ is a Ricci potential for $\omega_{h}$ for each fixed $\boldsymbol{\sigma}$, i.e., $d_{h} d_{h}^{c} \kappa_{h}=\rho_{h}$ where $\rho_{h}$ is the Ricci form of $\omega_{h}$. Then $\kappa=\kappa_{h}+$ $\frac{1}{2} \log \operatorname{det} G_{r s}$ is a Ricci potential for $g$, and we have

$$
\begin{aligned}
d_{h} d_{h}^{c} \kappa & =\rho_{h}+\frac{1}{2} d_{h}\left(H_{r s} d_{h}^{c} G_{r s}\right), \\
\frac{\partial \kappa}{\partial u_{r}} & =-\frac{1}{2 Q} \frac{\partial}{\partial \sigma_{t}}\left(Q H_{r t}\right) .
\end{aligned}
$$

Proof. (i) Expanding $d$ into horizontal and vertical parts, we get

$$
d d^{c} f=d_{h} d_{h}^{c} f+\frac{\partial f}{\partial u_{r}} d \theta_{r}+d \sigma_{r} \wedge \frac{\partial}{\partial \sigma_{r}} d_{h}^{c} f+d_{h} \frac{\partial f}{\partial u_{r}} \wedge \theta_{r}+\frac{\partial}{\partial \sigma_{s}} \frac{\partial f}{\partial u_{r}} d \sigma_{s} \wedge \theta_{r} .
$$

The second term in the equation (35) for $d \theta_{r}$ combines with the third term in the above equation to give $d_{h}^{c}\left(\partial f / \partial u_{r}\right) \wedge J \theta_{r}$. The formula for the laplacian (39) follows by contracting with $\omega$, using (37).

Now $d d^{c} f=0$ if and only if the three lines on the right hand side of (38) are separately zero. Hence $\partial f / \partial u_{r}$ must be constant, i.e., $f=$ $A_{r} u_{r}+B$ with $A_{r}$ constant and $\partial B / \partial u_{r}=0$, and so $d d^{c} f=0$ if and only if $d d^{c} B=d_{h} d_{h}^{c} B=0$.

(ii) A Ricci potential has the form $-\frac{1}{2} \log \left(\operatorname{vol}_{\omega} / \operatorname{vol}_{J}\right)$ where $\operatorname{vol}_{\omega}=$ $\frac{1}{m !} \omega \wedge \cdots \wedge \omega$ and $\operatorname{vol}_{J}$ is a holomorphic volume form. We first observe (see $[\mathbf{2 2}, \mathbf{2 6}])$ that if $d z_{\mu}$ is a local frame of holomorphic $(1,0)$-forms on $S$, then there are functions $B_{r \mu}$ such that $\sum_{\mu} B_{r \mu} d z_{\mu}+G_{r s} d \sigma_{s}+i \theta_{r}$, together with $d z_{\mu}$, form a local holomorphic frame of $M$. Since $\omega=$ $\omega_{h}+d \sigma_{r} \wedge \theta_{r}$, the formula for $\kappa$ is immediate.

Equation (40) follows easily using the fact that for any matrix valued function $A, d \log \operatorname{det} A=\operatorname{tr} A^{-1} d A$. For (41) we also note that $\kappa_{h}=$ $-\frac{1}{2} \log \left(Q \operatorname{vol}_{S} / \operatorname{vol}_{J_{h}}\right)$, where $\operatorname{vol}_{J_{h}}$ is a $(\boldsymbol{\sigma}$-independent) holomorphic 
volume form on $S$, and so

$$
\begin{aligned}
\frac{\partial \kappa}{\partial u_{r}} & =H_{r t} \frac{\partial \kappa}{\partial \sigma_{t}} \\
& =\frac{1}{2} H_{r t}\left(H_{p q} \frac{\partial G_{p q}}{\partial \sigma_{t}}-\frac{1}{Q} \frac{\partial Q}{\partial \sigma_{t}}\right) \\
& =\frac{1}{2}\left(H_{r t} \frac{\partial G_{t p}}{\partial \sigma_{q}} H_{p q}-\frac{1}{Q} \frac{\partial Q}{\partial \sigma_{t}} H_{r t}\right) \\
& =-\frac{1}{2}\left(\frac{\partial H_{r t}}{\partial \sigma_{t}}+\frac{1}{Q} \frac{\partial Q}{\partial \sigma_{t}} H_{r t}\right),
\end{aligned}
$$

where we use the symmetry of $\partial G_{p q} / \partial \sigma_{t}$ in $p, q, t$.

q.e.d.

This result provides conditions for $M$ to be Kähler-Einstein, using the fact that invariant Ricci and Kähler potentials then differ by an invariant pluriharmonic function. More generally, substituting (40) and (41) into (38) and (39) gives the Ricci form and scalar curvature. These expressions are rather complicated in general. However, if we suppose that $d_{h} G_{r s}=0$ and $d_{h} Q=0$ then

$$
\begin{aligned}
\rho & =\rho_{h}-\frac{1}{2 Q} \frac{\partial\left(Q H_{r t}\right)}{\partial \sigma_{t}} \omega_{h}-\frac{\partial}{\partial \sigma_{s}}\left(\frac{1}{2 Q} \frac{\partial\left(Q H_{r t}\right)}{\partial \sigma_{t}}\right) d \sigma_{s} \wedge \theta_{r}, \\
\mathrm{Scal} & =\mathrm{Scal}_{h}-\frac{1}{Q} \frac{\partial^{2}}{\partial \sigma_{r} \partial \sigma_{s}}\left(Q H_{r s}\right) .
\end{aligned}
$$

Note that these expressions depend linearly in $H_{r s}$ : this fact was emphasised by Abreu [1] in the toric case, and by Hwang-Singer [15] in the case of circle symmetry: we have just combined their arguments. We shall see the significance of the conditions $d_{h} G_{r s}=0$ and $d_{h} Q=0$ shortly.

We remark that when $m=2, \ell=1$, the Pedersen-Poon construction reduces to LeBrun's construction $[\mathbf{1 9}]$ :

$$
g=w e^{u}\left(d x^{2}+d y^{2}\right)+w d z^{2}+w^{-1} \theta^{2}, \quad \omega=w e^{u} d x \wedge d y+d z \wedge \theta,
$$

where $w_{x x}+w_{y y}+\left(w e^{u}\right)_{z z}=0$, which is the integrability condition for $d \theta=w_{x} d y \wedge d z-w_{y} d x \wedge d z+\left(w e^{u}\right)_{z} d x \wedge d y$. Here $d_{h} w=w_{x} d x+w_{y} d y$. Note $\kappa=-\frac{1}{2} u$ is a Ricci potential for $g$.

3.2. Rigid hamiltonian torus actions. Kähler manifolds with a hamiltonian $\ell$-torus action are too complicated, in their fullest generality, for constructing interesting Kähler metrics. Indeed most applications, including those in [22], use only Kähler metrics in the following subclass.

Proposition 8. Suppose the Kähler manifold $(M, g, J, \omega)$ has a local (isometric) hamiltonian $\ell$-torus action $\boldsymbol{K}=J \operatorname{grad}_{g} \boldsymbol{\sigma}$, for $\boldsymbol{\sigma}: M \rightarrow$ $\mathbb{R}^{\ell *}$, and let $\mathcal{F}$ be the foliation generated by $K_{r}, J K_{r}(r=1, \ldots \ell)$. Then on the open dense set $M^{0}$, where the action is locally free, the following are equivalent: 
(i) the leaves of $\mathcal{F}$ are totally geodesic;

(ii) the connection $\boldsymbol{\theta}: T M^{0} \rightarrow \mathbb{R}^{\ell}$, with $\operatorname{ker} \boldsymbol{\theta}=(\operatorname{span} \boldsymbol{K})^{\perp}$, is $J \boldsymbol{K}$ invariant;

(iii) $\left\langle K_{r}, K_{s}\right\rangle$ is constant on the level surfaces of $\boldsymbol{\sigma}$ for all $r, s$;

(iv) the family of Kähler forms $\omega_{h}=\omega-d \sigma_{r} \wedge \theta_{r}$ on the local leaf space of $\mathcal{F}$ depends affinely on $\boldsymbol{\sigma}$ and the linear part pulls back to the curvature of $\boldsymbol{\theta}$;

(v) there is a (local) $\boldsymbol{K}$-invariant Kähler potential of the form $H=$ $H_{0}+H_{\sigma}$ where $H_{0}$ is constant on the leaves of $\mathcal{F}$ and $H_{\sigma}$ is constant on the level surfaces of $\boldsymbol{\sigma}$.

Proof. The conditions (i)-(iii) are all equivalent to the fact that $\left\langle\nabla_{K_{r}} K_{s}, X\right\rangle=0$ for all $r, s$ and all $X$ orthogonal to $\mathcal{F}$. Indeed, since $J$ is parallel and $K_{r}$ is holomorphic this says that $\mathcal{F}$ is totally geodesic. On the other hand, $\left\langle\mathcal{L}_{J K_{r}}(J X), K_{s}\right\rangle=\left\langle J \mathcal{L}_{J K_{r}}(X), K_{s}\right\rangle=\left\langle J \nabla_{J K_{r}} X+\right.$ $\left.\nabla_{X} K_{r}, K_{s}\right\rangle=-2\left\langle\nabla_{K_{r}} K_{s}, X\right\rangle$, so it also says that the connection $\boldsymbol{\theta}$ is $J K_{r}$-invariant. Finally, it means that $\partial_{X}\left\langle K_{r}, K_{s}\right\rangle=-2\left\langle\nabla_{K_{r}} K_{s}, X\right\rangle=0$ for all $X$ orthogonal to $\mathcal{F}$.

To establish the equivalence of the local conditions (iii)-(v), we use the Pedersen-Poon construction, Proposition 6. (iii) means that $d_{h} H_{r s}$ $=0$, or equivalently $d_{h} G_{r s}=0$, which by (35) is equivalent to $d \theta_{r}=$ $\partial \omega_{h} / \partial \sigma_{r}$; this is (iv), since (34) then shows that $\omega_{h}$ is affine in $\boldsymbol{\sigma}$. (v) gives that $H_{r s}$ is the hessian of $H_{\sigma}$, which implies (iii). Conversely (iii) implies that the dual potential $G$ is an affine function of $\boldsymbol{\sigma}$, so that $d_{h} H=\sigma_{r}\left(\partial / \partial \sigma_{r}\right)\left(d_{h} G\right)-d_{h} G$ is independent of $\boldsymbol{\sigma}$; then it has a local $\boldsymbol{\sigma}$-independent $d_{h}$-potential $H_{0}$, and $d_{h} H_{\sigma}=0$ where $H_{\sigma}=H-H_{0}$.

q.e.d.

If $M$ is given by the Pedersen-Poon construction (as it is locally), then (i) means that the fibres of $M \rightarrow S$ (the complex orbits) are totally geodesic, (ii) that $M \rightarrow B$ is the pullback of a principal bundle with connection over $S$, and (iii) that the metric on the fibres of $M \rightarrow B$ (the torus orbits) depends only on the momentum map; the condition (iv) on the Kähler quotient is a kind of rigid Duistermaat-Heckman property (it holds in cohomology by [12]), while (v) generalizes Calabi's Ansatz [9] for Kähler metrics on holomorphic bundles.

Definition 4. A local hamiltonian $\ell$-torus action $\boldsymbol{K}=J \operatorname{grad}_{g} \boldsymbol{\sigma}$ on a Kähler manifold will be called rigid if $\left\langle K_{r}, K_{s}\right\rangle$ is constant on the level surfaces of $\sigma$.

Proposition 9. Suppose that $M$ arises from the Pedersen-Poon construction for a rigid hamiltonian $\ell$-torus action, and let $\nabla^{\|}$and $\nabla^{H}$ be respectively the Levi-Civita connection on the fibres of $M$ over $S$, and the Levi-Civita connection on the level surfaces of $\boldsymbol{\sigma}$ in B, lifted to the 
horizontal distribution of $M \rightarrow S$. Let $X, Y$ be horizontal vector fields and $U, V$ be vertical vector fields. Then

$$
\begin{aligned}
& \nabla_{X} Y=\nabla_{X}^{H} Y-C(X, Y) \\
& \nabla_{X} U=\langle C(X, \cdot), U\rangle+[X, U]^{\|} \\
& \nabla_{U} X=[U, X]^{H}+\langle C(X, \cdot), U\rangle \\
& \nabla_{U} V=\nabla_{U}^{\|} V,
\end{aligned}
$$

where ${ }^{H}$ and $\|$ denote the horizontal and vertical components, and the $O$ 'Neill tensor $C$ is given by

$$
2 C(X, Y)=\Omega_{r}(X, Y) K_{r}+\Omega_{r}(J X, Y) J K_{r} .
$$

Proof. These observations all follow from the Koszul formula

$$
\begin{aligned}
2\left\langle\nabla_{X} Y, Z\right\rangle= & \partial_{X}\langle Y, Z\rangle+\partial_{Y}\langle X, Z\rangle-\partial_{Z}\langle X, Y\rangle \\
& +\langle[X, Y], Z\rangle-\langle[X, Z], Y\rangle-\langle[Y, Z], X\rangle .
\end{aligned}
$$

The contraction of (42) with a horizontal vector field $Z$ is immediate because $M \rightarrow B$ is a riemannian submersion. For the vertical component, (47) gives

$$
2\left\langle\nabla_{X} Y, Z\right\rangle=\left(\mathcal{L}_{Z} g\right)(X, Y)+\langle[X, Y], Z\rangle
$$

(with $Z$ vertical). Taking $Z=\partial / \partial \sigma_{r}$ and $Z=K_{r}$ we obtain (42) with $C$ given by $(46)$, since $[X, Y]^{\|}=-\Omega_{r}(X, Y) K_{r}, J K_{r}=-\operatorname{grad}_{g} \sigma_{r}$, and

$$
\frac{\partial g}{\partial \sigma_{r}}(X, Y)=-\frac{\partial \omega_{h}}{\partial \sigma_{r}}(J X, Y)=-\Omega_{r}(J X, Y) .
$$

The remaining three equations are much easier: $\left\langle\nabla_{X} U, Y\right\rangle=-\left\langle\nabla_{X} Y, U\right\rangle$, $\nabla_{U} X-\nabla_{X} U=[U, X]$ and $\left\langle\nabla_{U} X, V\right\rangle=-\left\langle\nabla_{U} V, X\right\rangle$, so we only need to check $\left\langle\nabla_{X} U, V\right\rangle=\langle[X, U], V\rangle$ and (45). These follow immediately because the metric on the fibres is constant along horizontal curves and the fibres are totally geodesic.

q.e.d.

\subsection{Semisimple Kähler quotients.}

Definition 5. A complex manifold $(S, J)$ with a family of Kähler metrics $\left(h, \omega_{h}\right)$ (with parameter $\boldsymbol{\sigma}$ ) is semisimple if there is a Kähler form $\Omega_{S}$ on $S$ with respect to which the $\omega_{h}$ are simultaneously diagonalizable and parallel. A local hamiltonian torus action is semisimple if its local Kähler quotient is.

We can of course take $\Omega_{S}$ to be $\omega_{h}$ for some fixed $\boldsymbol{\sigma}$, but it will be convenient later to make a different choice.

Proposition 10. If $(S, J)$ is semisimple then $\left(S, \Omega_{S}\right)$ is locally a Kähler product of $\left(S_{a}, \omega_{a}\right)(a=1, \ldots N, N \geq 1)$ such that $\omega_{h}=$ $\sum_{a=1}^{N} c_{a}(\boldsymbol{\sigma}) \omega_{a}$, where $c_{a}(\boldsymbol{\sigma})$ is constant on $S$. The Levi-Civita connection of $\omega_{h}$ is independent of $\boldsymbol{\sigma}$, being equal to the Levi-Civita connection of $\Omega_{S}$. 
Proof. As $\omega_{h}$ is a parallel $(1,1)$-form on $S$, the ( $J$-invariant, simultaneous) eigendistributions of $\omega_{h}$ are parallel, and $S$ splits as a local Kähler product by the deRham theorem. The Levi-Civita connections of $\omega_{h}$ and $\Omega_{S}$ agree, because on each factor $S_{a}$ of the local Kähler product they are related by a constant multiple.

q.e.d.

In the case of local hamiltonian $\ell$-torus actions, the semisimplicity condition implies in particular that the quantity $Q$ defined by $\operatorname{vol}_{\omega_{h}}=$ $Q \operatorname{vol}_{S}$, where $\operatorname{vol}_{S}$ is the volume form of $\Omega_{S}$, is constant on $S$, being given by

$$
Q=\prod_{a=1}^{N} c_{a}(\boldsymbol{\sigma})^{m_{a}}, \quad \text { where } \quad \operatorname{dim} S_{a}=2 m_{a} .
$$

If the action is also rigid, $\omega_{h}$ depends affinely on $\sigma \in \mathbb{R}^{\ell *}$, so we can write $\omega_{h}=\Omega_{0}+\langle\boldsymbol{\sigma}, \boldsymbol{\Omega}\rangle$ where $\Omega_{0}$ and $\boldsymbol{\Omega}$ are closed $J$-invariant $(\boldsymbol{\sigma}$ independent) 2 -forms on $S$, the latter with values in $\mathbb{R}^{\ell}$. Letting $\Omega_{r}$ denote the components of $\boldsymbol{\Omega}$, we have $\Omega_{r}=\sum_{a=1}^{N} c_{a r} \omega_{a}$ for $r=0, \ldots \ell$, where $c_{a}(\boldsymbol{\sigma})=c_{a 0}+c_{a r} \sigma_{r}$.

\subsection{Orthotoric Kähler metrics.}

Definition 6. A Kähler $2 m$-manifold $(M, g, J, \omega)$ is orthotoric if it is equipped with $m$ Poisson-commuting functions $\sigma_{1}, \ldots \sigma_{m}$ such that $K_{r}=\operatorname{Jgrad}_{g} \sigma_{r}$ are Killing vector fields and, on a dense open set $M^{0}$, the roots $\xi_{j}$ of $\sum_{r=0}^{m}(-1)^{r} \sigma_{r} t^{\ell-r}\left(\sigma_{0}=1\right)$ are smoothly defined, with linearly independent, orthogonal gradients.

Note that an orthotoric Kähler manifold is toric, and any toric Riemann surface is orthotoric. For a higher dimensional toric manifold it is hard to detect whether it is orthotoric, since the condition depends on a choice of basis for Lie algebra of the torus. Because of this choice, we abandon the summation convention.

The exterior derivative of the identity

$$
\prod_{k=1}^{m}\left(t-\xi_{k}\right)=\sum_{r=0}^{m}(-1)^{r} \sigma_{r} t^{m-r}
$$

at $t=\xi_{j}$ yields

$$
d \xi_{j}=\frac{1}{\Delta_{j}} \sum_{r=0}^{m}(-1)^{r-1} \xi_{j}^{m-r} d \sigma_{r},
$$

where $\Delta_{j}=\prod_{k \neq j}\left(\xi_{j}-\xi_{k}\right)$. This is inverse to the identity

$$
d \sigma_{r}=\sum_{j=1}^{m} \sigma_{r-1}\left(\hat{\xi}_{j}\right) d \xi_{j},
$$

where $\sigma_{r-1}\left(\hat{\xi}_{j}\right)$ denote the elementary symmetric functions of the $m-1$ functions $\xi_{k}$ with $\xi_{j}$ deleted (with the convention that $\sigma_{0}=1$ ). Hence 
the coordinate systems given by $\xi_{j}$ and $\sigma_{r}$ are related by the Vandermonde matrix and its inverse. We have collected some Vandermonde identities that we need in Appendix B.

Proposition 11. Let $(M, g, J, \omega)$ be an orthotoric Kähler $2 m$-manifold. Then, on any simply connected domain $U$ in $M^{0}$, there are $m$ functions $t_{r}$, each determined up to an additive constant, and $m$ functions $\Theta_{j}$ of one variable, such that $\left\{\xi_{1}, \ldots \xi_{m}, t_{1}, \ldots t_{m}\right\}$ form a coordinate system with respect to which the Kähler structure may be written

$$
\begin{aligned}
g & =\sum_{j=1}^{m} \frac{\Delta_{j}}{\Theta_{j}\left(\xi_{j}\right)} d \xi_{j}^{2}+\sum_{j=1}^{m} \frac{\Theta_{j}\left(\xi_{j}\right)}{\Delta_{j}}\left(\sum_{r=1}^{m} \sigma_{r-1}\left(\hat{\xi}_{j}\right) d t_{r}\right)^{2}, \\
\omega & =\sum_{j=1}^{m} d \xi_{j} \wedge\left(\sum_{r=1}^{m} \sigma_{r-1}\left(\hat{\xi}_{j}\right) d t_{r}\right)=\sum_{r=1}^{m} d \sigma_{r} \wedge d t_{r}, \\
J d \xi_{j} & =\frac{\Theta_{j}\left(\xi_{j}\right)}{\Delta_{j}} \sum_{r=1}^{m} \sigma_{r-1}\left(\hat{\xi}_{j}\right) d t_{r}, \quad J d t_{r}=(-1)^{r} \sum_{j=1}^{m} \frac{\xi_{j}^{m-r}}{\Theta_{j}\left(\xi_{j}\right)} d \xi_{j} .
\end{aligned}
$$

Conversely, for any $m$ real functions $\Theta_{j}$ of one variable, the almosthermitian structure defined by (51) is Kähler and orthotoric with dual potential

$$
G=-\sum_{j=1}^{m} \int^{\xi_{j}} \frac{\prod_{k}\left(t-\xi_{k}\right)}{\Theta_{j}(t)} d t=-\sum_{j=1}^{m} \int^{\xi_{j}} \frac{\sum_{r=0}^{m}(-1)^{r} \sigma_{r} t^{m-r}}{\Theta_{j}(t)} d t
$$

and Kähler potential

$$
H=\sum_{j=1}^{m} \int^{\xi_{j}} \frac{t^{m}}{\Theta_{j}(t)} d t
$$

Proof. We apply Proposition 6 to obtain the local expression. The condition that the $\xi_{j}$ have orthogonal gradients means that

$$
H_{r s}=\sum_{j=1}^{m} \sigma_{r-1}\left(\hat{\xi}_{j}\right) \sigma_{s-1}\left(\hat{\xi}_{j}\right)\left|d \xi_{j}\right|^{2}
$$

and hence

$$
G_{r s}=\sum_{j=1}^{m} \frac{(-1)^{r+s} \xi_{j}^{m-r} \xi_{j}^{m-s}}{\Delta_{j}^{2}\left|d \xi_{j}\right|^{2}} .
$$

We set $\Theta_{j}=\Delta_{j}\left|d \xi_{j}\right|^{2}$ so that

$$
\sum_{s=1}^{m} G_{r s} d \sigma_{s}=\sum_{j=1}^{m} \frac{(-1)^{r-1} \xi_{j}^{m-r}}{\Theta_{j}} d \xi_{j} .
$$


If $G_{r s}$ is the hessian of a function $G$, this must be closed, i.e.,

$$
\xi_{j}^{m-r} \frac{\partial \Theta_{j}}{\partial \xi_{k}}=\xi_{k}^{m-r} \frac{\partial \Theta_{k}}{\partial \xi_{j}}
$$

for all $j, k, r=1, \ldots m$. Multiplying this by $(-1)^{r} d \sigma_{r}$ and using (49) to sum over $r$ (which amounts to inverting the above Vandermonde system) shows that $\Delta_{j} \partial \Theta_{j} / \partial \xi_{k}$ vanishes for $j \neq k$; it follows that each $\Theta_{j}$ only depends on $\xi_{j}$. Changing coordinates from Proposition 6 yields (51).

Conversely, if $\Theta_{j}$ is a function only of $\xi_{j}$ then (56) is equal to $d u_{r}$ where

$$
u_{r}=-\sum_{j=1}^{m} \int^{\xi_{j}} \frac{(-1)^{r} t^{m-r}}{\Theta_{j}(t)} d t .
$$

Since the integrand in (52) vanishes when $t=\xi_{j}$, the derivative of $G$ with respect to $\sigma_{r}$ is $u_{r}$, so its hessian $G_{r s} ; \sum_{r=1}^{m} u_{r} \sigma_{r}-G$ then gives (53). Since the $d \xi_{j}$ are evidently pairwise orthogonal, the structure is orthotoric.

q.e.d.

We end with an alternative characterization of orthotoric Kähler metrics.

Proposition 12. A toric Kähler structure $(g, J, \omega)$ is orthotoric if and only if there is a momentum map $\left(\sigma_{1}, \ldots \sigma_{m}\right)$ such that $\sum_{r=1}^{m}\left(\sigma_{1} \sigma_{r}-\right.$ $\left.\sigma_{r+1}\right) d u_{r}$ is a closed 1-form, where $u_{r}=\partial G / \partial \sigma_{r}$ for a dual potential $G$.

Proof. Let $\xi_{j}$ be the roots of the polynomial $\sum_{r=0}^{m}(-1)^{r} \sigma_{r} t^{m-r}$. Then

$$
\begin{aligned}
\sum_{r=1}^{m} d\left(\sigma_{1} \sigma_{r}-\sigma_{r+1}\right) \wedge d u_{r} & =\sum_{r, s} G_{r s}\left(\sigma_{r} d \sigma_{1}-d \sigma_{r+1}\right) \wedge d \sigma_{s} \\
& =\sum_{r, s, j, k} G_{r s}\left(\xi_{j} \sigma_{r-1}\left(\hat{\xi}_{j}\right) d \xi_{j}\right) \wedge\left(\sigma_{s-1}\left(\hat{\xi}_{k}\right) d \xi_{k}\right),
\end{aligned}
$$

which is zero if and only if $\sum_{r, s} G_{r s}\left(\xi_{j}-\xi_{k}\right) \sigma_{r-1}\left(\hat{\xi}_{j}\right) \sigma_{s-1}\left(\hat{\xi}_{k}\right)=0$ for all $j, k$. The left hand side is $\left(\xi_{j}-\xi_{k}\right)\left\langle\partial / \partial \xi_{j}, \partial / \partial \xi_{k}\right\rangle$, so the result follows.

q.e.d.

\section{Classification of hamiltonian 2-forms}

4.1. Rough classification of hamiltonian 2-forms. On any Kähler manifold, any $J$-invariant parallel 2 -form is hamiltonian. However, in this case the Killing vector fields $K(t)$ are all identically zero. For a general hamiltonian 2-form $\phi$, it is important to know how many of the $K(t)$ are linearly independent. To do this, we (temporarily) write $p(t)=\sum_{r=0}^{m}(-1)^{r} \sigma_{r} t^{m-r}$ and $K_{r}=J \operatorname{grad}_{g} \sigma_{r}$ for the coefficients of the momentum polynomial and corresponding Killing vector fields. Hence $K(t)=\sum_{r=1}^{m}(-1)^{r} K_{r} t^{m-r}$ is a linear combination of $K_{1}, \ldots K_{m}$ for any $t$. 
Proposition 13. Let $\phi$ be a hamiltonian 2-form on a (connected) Kähler $2 m$-manifold $M$. Then there is an integer $\ell$, with $0 \leq \ell \leq$ $m$ such that $K_{1} \wedge \cdots \wedge K_{\ell}$ is nonzero on a dense open subset, but $\operatorname{dim} \operatorname{span}\left\{K_{1}, \ldots K_{m}\right\} \leq \ell$ on all of $M$.

Proof. The coefficient of $t^{m-r}$ in identity (19) gives

$$
K_{r+1}=\phi\left(J K_{r}\right)+\sigma_{r} K_{1} .
$$

Suppose for some $z \in M$ and $1<s<m, K_{s}$ is a linear combination of $K_{1}, \ldots K_{s-1}$ at $z$. Then $K_{s+1}$ is also a linear combination of $K_{1}, \ldots K_{s-1}$ at $z$ : to see this, use (58) with $r=s$, then write $\phi\left(J K_{s}\right)$ as a linear combination of $\phi\left(J K_{r}\right)$ with $r<s$ and use (58) again to express these in terms of $K_{1}, \ldots K_{s}$. Hence, at each $z \in M$, dim $\operatorname{span}\left\{K_{1}, \ldots K_{m}\right\}$ is the largest integer $\ell_{z}$ such that $K_{1}, \ldots K_{\ell_{z}}$ are linearly independent at $z$. However, for any integer $r, K_{1}, \ldots K_{r}$ are linearly dependent if and only if the holomorphic $r$-vector $K_{1}^{1,0} \wedge \cdots \wedge K_{r}^{1,0}$ is zero. Hence the set where $K_{1}, \ldots K_{r}$ are linearly independent is empty or dense. The result follows.

q.e.d.

The integer $\ell$ of this proposition will be called the order of $\phi$ and we let $M^{0}$ be the dense open set where $K_{1}, \ldots K_{\ell}$ are independent. We shall identify the order of $\phi$ with the number of non-constant roots of the momentum polynomial $p$.

Lemma 3. If, on an open subset of $M, \phi(Z, \cdot)=\xi \omega(Z, \cdot)$ with $Z$ nonvanishing, then $d \xi$ is the orthogonal projection of $d \sigma$ onto the span of $Z$ and $J Z$.

Proof. Without loss of generality, we can take $Z$ to be a unit vector field, and hence $\left(\nabla_{X} \phi\right)(Z, J Z)=d \xi(X)$ for all vector fields $X$. By (12), this becomes

$$
d \xi=d \sigma(Z) Z+d \sigma(J Z) J Z,
$$

which is what we wanted to prove.

q.e.d.

The roots of the momentum polynomial are the eigenvalues of $-J \phi=$ $-J \circ \phi$, viewed as a $J$-commuting symmetric endomorphism of $T M$. At each point of $M$, these eigenvalues are real and there is an orthogonal $J$-invariant direct sum decomposition of the tangent space into eigenspaces. We count an eigenvalue with multiplicity $k$ if the corresponding eigenspace has real dimension $2 k$; for the moment, we denote by $\xi_{1}, \ldots \xi_{m}$ the $m$ (not necessarily distinct) eigenvalues of $-J \phi$. It follows that, for any $t$,

$$
p(t)=\prod_{j=1}^{m}\left(t-\xi_{j}\right)=t^{m}-\sigma_{1} t^{m-1}+\cdots+(-1)^{m} \sigma_{m},
$$


where $\sigma_{1}, \ldots \sigma_{m}$ are the elementary symmetric functions of $\xi_{1}, \ldots \xi_{m}$. The above lemma, and the independence of $K_{1}, \ldots K_{\ell}$ on $M^{0}$, yields a fundamental fact.

Proposition 14. Let $\phi$ be a hamiltonian 2-form on $M$. Then the roots $\xi_{i}$ of $p(t)$ and their derivatives $d \xi_{i}$ may be defined smoothly on the dense open set $M^{0}$ and the roots extend continuously to $M$. Furthermore, for $i \neq j, d \xi_{i}$ and $d \xi_{j}$ are orthogonal on $M$. In particular, any repeated root (on an open set) is constant.

If the order of $\phi$ is $\ell$, then there are $\ell$ non-constant roots and they are functionally independent on the dense open set where $K_{1}, \ldots K_{\ell}$ are independent.

Proof. The ordered roots $\xi_{1} \leq \cdots \leq \xi_{m}$ are continuous on $M$. By the maximality of $\ell$, wherever $\xi_{1}, \ldots \xi_{m}$ can be smoothly defined, at most $\ell$ of the $d \xi_{i}$ are independent. On the other hand, on any open subset of $M^{0}$ where $\xi_{1}, \ldots \xi_{m}$ are smoothly defined, at least $\ell$ of the $d \xi_{i}$ are independent. It follows that the $\xi_{i}$ and $d \xi_{i}$ can be defined smoothly on $M^{0}$, and that precisely $\ell$ of the $\xi_{i}$ are functionally independent there. Lemma 3 now shows that for $i \neq j, d \xi_{i}$ and $d \xi_{j}$ are orthogonal. q.e.d.

The order expresses the extent to which $\phi$ constrains the Kähler geometry of $M$. At one extreme, when $\ell=0$, we have an orthogonal $J$-invariant decomposition of $T M$ into eigenspaces of $-J \phi$, and it is easy to see that this makes $M$ into a local Kähler product: if we write $\omega=\sum_{\xi} \omega_{\xi}$ and $\phi=\sum_{\xi} \xi \omega_{\xi}$ where $\omega_{\xi}$ is the restriction of the Kähler form to the $\xi$ eigenspace, then the closedness of $\phi$ and $\omega$ is equivalent to $d \omega_{\xi}=0$ for each $\xi$-for instance there could be only one eigenspace (in which case $\phi$ is a constant multiple of the Kähler form), or there could be $m$ (in which case $M$ is an arbitrary Kähler product of Riemann surfaces). At the other extreme, when $\ell=m, M$ is toric and Lemma 3 shows in fact that it is orthotoric, and so has the explicit form of Proposition 11, determined by $m$ functions of one variable.

4.2. Explicit description of the metric. We now present the general description of Kähler metrics with a hamiltonian 2-form. To do this, it will be convenient to adopt different notation from the previous subsection.

Definition 7. Let $p(t)=(-1)^{m} \operatorname{pf}(\phi-t \omega)$ be the momentum polynomial of a hamiltonian 2 -form $\phi$ of order $\ell$, and let $\xi_{1}, \ldots \xi_{\ell}$ the nonconstant roots of $p$. We denote by $\sigma_{0}, \ldots \sigma_{\ell}$ the elementary symmetric functions of these non-constant roots, set $K_{r}:=J \operatorname{grad}_{g} \sigma_{r}$ for $r=$ 
$1, \ldots \ell$, and write $p(t)=p_{\mathrm{c}}(t) p_{\mathrm{nc}}(t)$, where

$$
\begin{aligned}
p_{\mathrm{nc}}(t) & :=\prod_{j=1}^{\ell}\left(t-\xi_{j}\right)=t^{\ell}-\sigma_{1} t^{\ell-1}+\cdots+(-1)^{\ell} \sigma_{\ell}, \\
p_{\mathrm{c}}(t) & :=\prod_{\xi}(t-\xi)^{m_{\xi}},
\end{aligned}
$$

and the product over $\xi$ denotes the product over the different constant roots of $p(t), m_{\xi}$ being the multiplicity of the root $\xi$.

If we denote the $\sigma$ 's of the previous subsection by $\tilde{\sigma}_{1}, \ldots \tilde{\sigma}_{m}$, then for $\ell=m \tilde{\sigma}_{r}=\sigma_{r}$; otherwise, since the roots of $p_{\mathrm{c}}$ are constant, it follows that $d \tilde{\sigma}_{1}, \ldots d \tilde{\sigma}_{m}$ are constant linear combinations of $d \sigma_{1}, \ldots d \sigma_{\ell}$. Now $d \tilde{\sigma}_{1}, \ldots d \tilde{\sigma}_{\ell}$ are linearly independent. Hence $d \sigma_{1}, \ldots d \sigma_{\ell}$ are also linearly independent, and $\sigma_{1}, \ldots \sigma_{\ell}$ are constant affine linear combinations of $\tilde{\sigma}_{1}, \ldots \tilde{\sigma}_{\ell}$. (Note in particular that $d \sigma_{1}=d \tilde{\sigma}_{1}=d \sigma$.)

Hence $K_{1}, \ldots K_{\ell}$ generate a local hamiltonian $\ell$-torus action and $M^{0}$ is locally a bundle over the Kähler quotient $S$ with toric fibres. The tangent space $\mathcal{V}$ to the fibres of $M$ over $S$ is spanned by $K_{1}, \ldots K_{\ell}$ and $J K_{1}, \ldots J K_{\ell}$, while the orthogonal distribution $\mathcal{H}$ is the direct sum of the eigendistributions $\mathcal{H}_{\xi}$ corresponding to the constant eigenvalues $\xi$ of $-J \phi$. We let $\Omega$ be the the $\mathcal{V}$-valued 2 -form on $\mathcal{H}$ defined by $\Omega(Y, Z)=$ $[Y, Z]^{\mathcal{V}}$, the orthogonal projection of the Lie bracket onto $\mathcal{V}$.

\section{Lemma 4.}

(i) For all $\xi$ and $r$, the distribution $\mathcal{H}_{\xi}$ is $K_{r}$ and $J K_{r}$ invariant, and descends to a parallel distribution on $S$ (with respect to each quotient metric).

(ii) If $Y$ and $Z$ belong to $\mathcal{H}_{\xi}$ and $\mathcal{H}_{\eta}$ for distinct constant eigenvalues $\xi \neq \eta$ then $\Omega(Y, Z)=0$. If instead $Y$ and $Z$ both belong to $\mathcal{H}_{\xi}$, then

$$
\Omega(Y, Z)=\frac{\omega(Y, Z)}{p_{\mathrm{nc}}(\xi)} \sum_{r=1}^{\ell}(-1)^{r-1} \xi^{\ell-r} K_{r} .
$$

Proof. Suppose that $\phi(Z)=\xi J Z$ for a constant root $\xi$. Then

$\phi\left(\nabla_{Y} Z\right)=\nabla_{Y}(\xi J Z)-\left(\nabla_{Y} \phi\right)(Z)=\xi J \nabla_{Y} Z-\frac{1}{2} \iota_{Z}\left(d \sigma_{1} \wedge J Y-d^{c} \sigma_{1} \wedge Y\right)$

for any vector field $Y$, and hence

$$
2(\phi-\xi J)\left(\nabla_{Y} Z\right)=\omega(Y, Z) d \sigma_{1}-\langle Y, Z\rangle J d \sigma_{1}
$$

since $d \sigma_{1}(Z)=0=J d \sigma_{1}(Z)$ (cf. Lemma 3 and equation (59)).

(i) We apply (62) with $Z$ in $\mathcal{H}_{\xi}$ and $Y$ orthogonal to $\mathcal{H}_{\xi}$ to deduce that $\nabla_{Y} Z$ also belongs to $\mathcal{H}_{\xi}$. Now $\mathcal{L}_{Y} Z=\nabla_{Y} Z-\nabla_{Z} Y$, and the first term is in $\mathcal{H}_{\xi}$ for $Y=K_{r}$ or $J K_{r}$. On the other hand, for $X$ orthogonal to $\mathcal{H}_{\xi},\left\langle\nabla_{Z} J K_{r}, J X\right\rangle=\left\langle\nabla_{Z} K_{r}, X\right\rangle=-\left\langle\nabla_{X} K_{r}, Z\right\rangle=\left\langle K_{r}, \nabla_{X} Z\right\rangle=0$, so that $\nabla_{Z} Y$ is also in $\mathcal{H}_{\xi}$ for $Y=K_{r}$ or $J K_{r}$. Thus $\mathcal{H}_{\xi}$ descends to 
$S$, and since the Levi-Civita connections of the Kähler quotient metrics lift to the horizontal $(\mathcal{H})$ part of $\nabla$, this distribution is parallel by $(62)$.

(ii) This again follows from (62): if $Y$ and $Z$ belong to distinct eigenspaces, then $\omega(Y, Z)=\langle Y, Z\rangle=0$, so that $[Y, Z]$ is in $\mathcal{H}$; otherwise, if they both belong the the $\xi$ eigenspace, we have

$$
\begin{aligned}
(\phi-\xi J)([Y, Z]) & =\omega(Y, Z) d \sigma_{1}=\omega(Y, Z) \sum_{j=1}^{\ell} d \xi_{j} \\
& =\omega(Y, Z) \sum_{j=1}^{\ell} \frac{(\phi-\xi J) J d \xi_{j}}{\xi-\xi_{j}} .
\end{aligned}
$$

Hence

$$
\begin{aligned}
\Omega(Y, Z) & =\omega(Y, Z) \sum_{j=1}^{\ell} \frac{J d \xi_{j}^{\sharp}}{\xi-\xi_{j}} \\
& =\frac{\omega(Y, Z)}{\prod_{k=1}^{\ell}\left(\xi-\xi_{k}\right)} \sum_{j=1}^{\ell}\left(\prod_{k \neq j}\left(\xi-\xi_{k}\right)\right) J d \xi_{j}^{\sharp}
\end{aligned}
$$

from which (61) easily follows, since $K_{r}=J d \sigma_{r}^{\sharp}=\sum_{j=1}^{\ell} \sigma_{r-1}\left(\hat{\xi}_{j}\right) J d \xi_{j}^{\sharp}$. q.e.d.

This lemma, with Propositions 8, 11 and 14, yields our classification.

Theorem 1. Let $(M, g, J, \omega)$ be a connected Kähler $2 m$-manifold with a hamiltonian 2-form $\phi$ of order $\ell$. Then there are functions $F_{1}, \ldots F_{\ell}$ of one variable such that on a dense open subset $M^{0}$, the Kähler structure may be written

$$
\begin{aligned}
g & =\sum_{\xi} p_{\mathrm{nc}}(\xi) g_{\xi}+\sum_{j=1}^{\ell} \frac{p^{\prime}\left(\xi_{j}\right)}{F_{j}\left(\xi_{j}\right)} d \xi_{j}^{2}+\sum_{j=1}^{\ell} \frac{F_{j}\left(\xi_{j}\right)}{p^{\prime}\left(\xi_{j}\right)}\left(\sum_{r=1}^{\ell} \sigma_{r-1}\left(\hat{\xi}_{j}\right) \theta_{r}\right)^{2}, \\
\omega & =\sum_{\xi} p_{\mathrm{nc}}(\xi) \omega_{\xi}+\sum_{r=1}^{\ell} d \sigma_{r} \wedge \theta_{r}, \quad d \theta_{r}=\sum_{\xi}(-1)^{r} \xi^{\ell-r} \omega_{\xi}, \\
J d \xi_{j} & =\frac{F_{j}\left(\xi_{j}\right)}{p^{\prime}\left(\xi_{j}\right)} \sum_{r=1}^{\ell} \sigma_{r-1}\left(\hat{\xi}_{j}\right) \theta_{r}, \quad J \theta_{r}=(-1)^{r} \sum_{j=1}^{\ell} \frac{p_{\mathrm{c}}\left(\xi_{j}\right)}{F_{j}\left(\xi_{j}\right)} \xi_{j}^{\ell-r} d \xi_{j}
\end{aligned}
$$

where summation over $\xi$ denotes the sum over the different constant roots of $p(t), \sigma_{r-1}\left(\hat{\xi}_{j}\right)$ denote the elementary symmetric functions of the $\ell-1$ functions $\xi_{k}$ with $\xi_{j}$ deleted, $p^{\prime}(t)$ is the derivative of the momentum polynomial $p(t)$ with respect to $t$, and $\pm\left(g_{\xi}, \omega_{\xi}\right)$ is a Kähler metric on a 
manifold $S_{\xi}$ of the same dimension as the $\xi$-eigenspace of $-J \phi$. Dual and Kähler potentials for $(g, J, \omega)$ are given by

$$
\begin{aligned}
& G=-\sum_{r=0}^{\ell} H_{r} \sigma_{r}-\sum_{j=1}^{\ell} \int^{\xi_{j}} \frac{p(t)}{F_{j}(t)} d t, \\
& H=H_{0}+\sum_{j=1}^{\ell} \int^{\xi_{j}} \frac{p_{\mathrm{c}}(t) t^{\ell}}{F_{j}(t)} d t,
\end{aligned}
$$

where $H_{r}$ is a $\left(\boldsymbol{\sigma}\right.$-independent) $d d^{c}$ potential for $\Omega_{r}=\sum_{\xi}(-1)^{r} \xi^{\ell-r} \omega_{\xi}$.

Furthermore, in these coordinates, the hamiltonian 2-form may be written

$$
\begin{aligned}
\phi & =\sum_{\xi} \xi p_{\mathrm{nc}}(\xi) \omega_{\xi}+\sum_{j=1}^{\ell} \xi_{j} d \xi_{j} \wedge\left(\sum_{r=1}^{\ell} \sigma_{r-1}\left(\hat{\xi}_{j}\right) \theta_{r}\right) \\
& =\sum_{\xi} \sum_{r=0}^{\ell}(-1)^{r} \sigma_{r} \xi^{\ell+1-r} \omega_{\xi}+\sum_{r=1}^{\ell}\left(\sigma_{r} d \sigma_{1}-d \sigma_{r+1}\right) \wedge \theta_{r} .
\end{aligned}
$$

We also have a local $d d^{c}$ potential for the closed form $\phi+\sigma_{1} \omega$ :

$$
\phi+\sigma_{1} \omega=\sum_{\xi} \xi^{\ell+1} \omega_{\xi}+\sum_{r=1}^{\ell} d\left(\left(\sigma_{1} \sigma_{r}-\sigma_{r+1}\right) \theta_{r}\right)=d d^{c} \Phi
$$

where

$$
\Phi=-H_{-1}+\sum_{j=1}^{\ell} \int^{\xi_{j}} \frac{p_{\mathrm{c}}(t) t^{\ell+1}}{F_{j}(t)} d t
$$

and $H_{-1}$ is a $(\boldsymbol{\sigma}$-independent $) d d^{c}$ potential for $\Omega_{-1}=-\sum_{\xi} \xi^{\ell+1} \omega_{\xi}$.

Proof. By Lemma 4, the distribution $\mathcal{H}$ is preserved by $J K_{1}, \ldots J K_{\ell}$; hence, by Proposition 8, the local fibration of $M^{0}$ over $S$ is totally geodesic, and the toric structure on the fibres is constant on the level surfaces of $\boldsymbol{\sigma}$. Therefore, the restriction of $\phi$ to any fibre is hamiltonian (even when $\ell=1$ since the trace of $\phi$ is a hamiltonian for a Killing vector field tangent to the fibres). By Proposition 14, the fibres are orthotoric, and the functions $G_{r s}$ are independent of the fibre, hence so are the functions $\Theta_{j}=\Delta_{j}\left|d \xi_{j}\right|^{2}$ defining the orthotoric structure in Proposition 11.

Again using Lemma 4, for the constant eigenvalues $\xi, \mathcal{H}_{\xi}$ descends to a $J$-invariant distribution on $S$, and $T S$ is the direct sum of these distributions. For each fixed value of $\boldsymbol{\sigma}$, these distributions are parallel with respect to the Kähler quotient metric $\left(h, \omega_{h}\right)$ and so $S$ splits locally as a Kähler product of manifolds $S_{\xi}$. Furthermore, the curvature $\Omega$ of $\mathcal{H}$ descends to $S$, so that the 2 -form $\omega_{\xi}=\omega / p_{\text {nc }}(\xi)$, appearing in the formula (61), descends to give a Kähler structure on $S_{\xi}$, after restricting 
it to the $\xi$-eigenspace distribution. (Note, however, that this Kähler structure will be negative definite if $p_{\mathrm{nc}}(\xi)$ is negative.)

Clearly $\omega_{h}=\sum_{\xi} p_{\text {nc }}(\xi) \omega_{\xi}=\sum_{r=0}^{\ell} \sigma_{r} \Omega_{r}$ with

$$
\Omega_{r}=\sum_{\xi}(-1)^{r} \xi^{\ell-r} \omega_{\xi}
$$

$(r=0, \ldots \ell)$. The explicit form of the metric on $M^{0}$ easily follows: we define $F_{j}(t)=p_{\mathrm{c}}(t) \Theta_{j}(t)$, and observe that $p^{\prime}\left(\xi_{j}\right)=p_{\mathrm{c}}\left(\xi_{j}\right) \Delta_{j}$, since $\Delta_{j}=\prod_{k \neq j}\left(\xi_{j}-\xi_{k}\right)$.

It remains to establish the explicit form of the potentials: observe that

$$
\omega=\Omega_{0}+\sum_{r=1}^{\ell} d\left(\sigma_{r} \theta_{r}\right)=d d^{c} H_{0}+\sum_{j=1}^{\ell} d J\left(\frac{\xi_{j}^{\ell} p_{\mathrm{c}}\left(\xi_{j}\right) d \xi_{j}}{F_{j}\left(\xi_{j}\right)}\right)=d d^{c} H .
$$

The equation $H=\sum_{r=1}^{\ell} \sigma_{r} \partial G / \partial \sigma_{r}-G$ determines $G$ up to a linear combination of the $\sigma_{r}$ with basic coefficients. We also require $d_{h} d_{h}^{c} G=$ $-\omega_{h}$ so the functions $u_{r}=\partial G / \partial \sigma_{r}$ are pluriharmonic; $G$ given by (64) has the required properties with

$$
u_{r}=-H_{r}-\sum_{j=1}^{\ell} \int^{\xi_{j}} \frac{(-1)^{r} p_{\mathrm{c}}(t) t^{\ell-r}}{F_{j}(t)} d t
$$

for $r=1, \ldots \ell$, where $H_{r}$ is a $d d^{c}$-potential for $\Omega_{r}$ on $S$.

In the formula for $\phi$, we have used the fact that $\xi_{j} \sigma_{r-1}\left(\hat{\xi}_{j}\right)=\sigma_{r}-$ $\sigma_{r}\left(\hat{\xi}_{j}\right)$. It is then straightforward to check that $\Phi$ is a $d d^{c}$ potential for $\phi+\sigma_{1} \omega$.

q.e.d.

Remark 5. Formally, we set $u_{0}=-H$, so that $G=\sum_{r=1}^{\ell} \sigma_{r} u_{r}-H=$ $\sum_{r=0}^{\ell} \sigma_{r} u_{r}$. Similarly, we can write $u_{-1}=\Phi$, and in general extend (69)(70) to all $r \leq \ell$, where $d d^{c} H_{r}=\Omega_{r}$. For $r>0 d d^{c} u_{r}=0$, while for $r=-k \leq 0$ we have $d d^{c} u_{-k}=(-1)^{k+1} \phi_{k}$ where

$$
\begin{aligned}
\phi_{k}= & \sum_{\xi} \xi^{\ell+k} \omega_{\xi}+\sum_{j, r=1}^{\ell} d\left(\frac{\left.\xi_{j}^{\ell+k} \sigma_{r-1}\right)\left(\hat{\xi}_{j}\right)}{\Delta_{j}} J d \xi_{j}\right) \\
= & \sum_{\xi}\left(\frac{\xi^{\ell+k}}{\prod_{k=1}^{\ell}\left(\xi-\xi_{k}\right)}+\sum_{j=1}^{\ell} \frac{\xi_{j}^{\ell+k}}{\Delta_{j}\left(\xi_{j}-\xi\right)}\right) p_{\mathrm{nc}}(\xi) \omega_{\xi} \\
& +\sum_{i, j, r=1}^{\ell} \frac{\partial}{\partial \xi_{i}}\left(\frac{\xi_{j}^{\ell+k} \sigma_{r-1}\left(\hat{\xi}_{j}\right)}{\Delta_{j}}\right) d \xi_{i} \wedge \theta_{r} .
\end{aligned}
$$

Using (95) and (97) from Appendix B, this may be written

$$
-J \phi_{k}=\sum_{s=0}^{k} h_{k-s}(-J \phi)^{s}
$$


and $h_{p}$ is the $p$ th complete symmetric function in $\xi_{1}, \ldots \xi_{\ell}$.

4.3. The hamiltonian 2-form. In order to complete the classification of Kähler metrics with a hamiltonian 2-form, we must show that the explicit metric of Theorem 1 actually admits a hamiltonian 2-form with no further constraints.

Theorem 2. Let $(g, J, \omega)$ be a Kähler structure given explicitly by $(63)$, where $p(t)=p_{\mathrm{nc}}(t) p_{\mathrm{c}}(t)$ is a degree $m$ polynomial such that $p_{\mathrm{nc}}(t) \in C^{\infty}(M, \mathbb{R})$ has functionally independent roots $\xi_{1}, \ldots \xi_{\ell}, p_{\mathrm{c}}(t)$ and constant roots $\xi$ with multiplicities $\operatorname{dim} g_{\xi}$, and $F_{1}, \ldots F_{\ell}$ are functions of one variable. Then the 2 -form $\phi$ defined by (66) is a hamiltonian 2 -form of order $\ell$ with momentum polynomial $p(t)$.

Proof. Obviously (for the case $m=1$ ), the trace of $\phi$ is a Killing potential. Hence, in order to show that $\phi$ is hamiltonian, we must show that

$$
\nabla A=d \sigma_{1} \otimes \omega+\frac{1}{2}\left(d \sigma_{1} \wedge J-d^{c} \sigma_{1} \wedge \mathrm{Id}\right)
$$

where $A=\phi+\sigma_{1} \omega$. Since $A$ is manifestly closed, we only need to check the equation for $\left(\nabla_{X} A\right)(Y, Z),\left(\nabla_{X} A\right)(Y, U),\left(\nabla_{U} A\right)(V, X)$ and $\left(\nabla_{U} A\right)(V, W)$, where $X, Y, Z$ and $U, V, W$ are arbitrary horizontal and vertical vector fields respectively. Two of these equations are immediate: $\left(\nabla_{X} A\right)(Y, Z)=0$ and $\left(\nabla_{U} A\right)(V, X)=0$.

We next consider the equation for $\left(\nabla_{X} A\right)(Y, U)$, which reduces, using Proposition 9, to the equation

$$
2[\phi, C(X)]=\langle X, \cdot\rangle \otimes J d \sigma_{1}^{\sharp}-\omega(X, \cdot) \otimes d \sigma_{1}^{\sharp} .
$$

Here the left hand side is the commutator of $\phi$ with

$$
2 C(X)=\sum_{r=1}^{\ell}\left(\Omega_{r}(X) \otimes J d \sigma_{r}^{\sharp}-\Omega_{r}(J X) \otimes d \sigma_{r}^{\sharp}\right) .
$$

Decomposing into the eigenspaces of $-J \phi$, we compute that

$$
\begin{aligned}
2[\phi, C(X)]=- & \sum_{r, j=1}^{\ell} \sum_{\xi}(-1)^{r} \xi^{\ell-r} \sigma_{r-1}\left(\hat{\xi}_{j}\right)\left(\xi_{j}-\xi\right) \\
& \cdot\left(\omega_{\xi}(X) \otimes d \xi_{j}^{\sharp}+\omega_{\xi}(J X) \otimes J d \xi_{j}^{\sharp}\right) \\
=- & \sum_{j=1}^{\ell} \sum_{\xi}\left(\xi^{\ell}+\sum_{r=1}^{\ell}(-1)^{r} \xi^{\ell-r}\left(\xi_{j} \sigma_{r-1}\left(\hat{\xi}_{j}\right)+\sigma_{r}\left(\hat{\xi}_{j}\right)\right)\right) \\
& \cdot\left(\omega_{\xi}(X) \otimes d \xi_{j}^{\sharp}+\omega_{\xi}(J X) \otimes J d \xi_{j}^{\sharp}\right) \\
=- & \sum_{r=0}^{\ell} \sum_{\xi}(-1)^{r} \sigma_{r} \xi^{\ell-r}\left(\omega_{\xi}(X) \otimes d \sigma_{1}^{\sharp}+\omega_{\xi}(J X) \otimes J d \sigma_{1}^{\sharp}\right) .
\end{aligned}
$$

This proves (71), by the definition of $g$ and $\omega$. 
It remains to verify the equation for $\left(\nabla_{U} A\right)(V, W)$. Since the fibres are totally geodesic, this amounts to showing that $\phi$ is a hamiltonian 2form on the fibres. Hence it suffices to prove the result in the orthotoric case, when we have $\phi+\sigma_{1} \omega=\sum_{r} d\left(f_{r} d^{c} u_{r}\right)$, with $f_{r}=\sigma_{1} \sigma_{r}-\sigma_{r+1}$.

To do this we shall use only the fact that $\sum_{r} f_{r} d u_{r}=d \Phi$ is closedequivalently $A$ is $J$-invariant: $A\left(J K_{r}, K_{s}\right)=d f_{r}\left(J K_{s}\right)=d f_{s}\left(J K_{r}\right)=$ $A\left(J K_{s}, K_{r}\right)$, cf. Proposition 12. We define $\Phi_{r s}:=A\left(J K_{r}, K_{s}\right)$ and recall that $\left\langle K_{r}, K_{s}\right\rangle=H_{r s}$.

Since $A$ is closed and $J$-invariant, it suffices to check

$$
\begin{aligned}
\left(\nabla_{K_{r}} A\right)\left(J K_{s}, K_{t}\right)= & 0 \\
\left(\nabla_{J K_{r}} A\right)\left(J K_{s}, K_{t}\right)= & -d \sigma_{1}\left(J K_{r}\right)\left\langle K_{s}, K_{t}\right\rangle \\
& -\frac{1}{2} d \sigma_{1}\left(J K_{s}\right)\left\langle K_{r}, K_{t}\right\rangle \\
& -\frac{1}{2} d \sigma_{1}\left(J K_{t}\right)\left\langle K_{s}, K_{r}\right\rangle .
\end{aligned}
$$

Equation (72) follows immediately: the left hand side is

$$
K_{r} \cdot\left(A\left(J K_{s}, K_{t}\right)\right)-A\left(J \nabla_{K_{r}} K_{s}, K_{t}\right)-A\left(J K_{s}, \nabla_{K_{r}} K_{t}\right)
$$

and all three terms are zero here, since $A\left(J K_{s}, K_{t}\right)$ is $K_{r}$-invariant, $J \nabla_{K_{r}} K_{s}$ is a linear combination of the $K_{t}$ 's, and $A\left(K_{r}, K_{s}\right)=0$ for all $r, s$.

On the other hand, for equation (73) we have

$$
\left(\nabla_{J K_{r}} A\right)\left(J K_{s}, K_{t}\right)=J K_{r} \cdot\left(d f_{s}\left(J K_{t}\right)\right)+d f_{t}\left(\nabla_{K_{r}} K_{s}\right)+d f_{s}\left(\nabla_{K_{r}} K_{t}\right) .
$$

Now

$$
\nabla_{K_{r}} K_{s}=-\frac{1}{2} \operatorname{grad}\left\langle K_{r}, K_{s}\right\rangle=-\frac{1}{2} \sum_{p} \frac{\partial H_{r s}}{\partial u_{p}} d u_{p}^{\sharp}
$$

and $H_{r s}=\partial^{2} H / \partial u_{r} \partial u_{s}$, so (73) holds if and only if

$$
\begin{aligned}
& \frac{\partial \Phi_{s t}}{\partial u_{r}}-\frac{1}{2} \sum_{p, q} G_{p q}\left(\Phi_{t p} \frac{\partial H_{s q}}{\partial u_{r}}+\Phi_{s p} \frac{\partial H_{t q}}{\partial u_{r}}\right) \\
& =H_{1 r} H_{s t}+\frac{1}{2}\left(H_{1 s} H_{r t}+H_{1 t} H_{r s}\right) .
\end{aligned}
$$

This simplifies once we observe that $\Phi_{s t}=\sum_{p, q} G_{p q} \Phi_{t p} H_{s q}=$ $\sum_{p, q} G_{p q} \Phi_{s p} H_{t q}$, so that the product rule reduces the left hand side to

$$
\frac{1}{2} \sum_{p, q}\left(H_{s q} \frac{\partial}{\partial u_{r}}\left(G_{p q} \Phi_{t p}\right)+H_{t q} \frac{\partial}{\partial u_{r}}\left(G_{p q} \Phi_{s p}\right)\right) .
$$

Now we use the fact that $f_{s}=\sigma_{1} \sigma_{s}-\sigma_{s+1}$ and $\partial \sigma_{s} / \partial u_{r}=H_{r s}$ to deduce that

$$
\sum_{p} \frac{\partial}{\partial u_{r}}\left(G_{p q} \Phi_{t p}\right)=H_{r t} \delta_{1 q}+H_{1 r} \delta_{t q}
$$

Substituting this into (75) yields (74), and hence (73).

q.e.d. 


\section{The curvature of Kähler manifolds with a hamiltonian 2-form}

5.1. The Ricci potential and scalar curvature. In this section we compute the Ricci potential and scalar curvature for any Kähler manifold $(M, g, J, \omega)$ given by (63), using the formulae obtained in section 3 . In terms of the Vandermonde matrix $V_{r j}$ and its inverse $W_{j r}$ (see Appendix B), we have

$$
G_{r s}=\sum_{j=1}^{\ell} \frac{p_{\mathrm{c}}\left(\xi_{j}\right) V_{r j} V_{s j}}{F_{j}\left(\xi_{j}\right) \Delta_{j}}, \quad H_{r s}=\sum_{j=1}^{\ell} \frac{W_{j r} W_{j s} F_{j}\left(\xi_{j}\right) \Delta_{j}}{p_{\mathrm{c}}\left(\xi_{j}\right)},
$$

and hence, up to a sign, $\operatorname{det} G_{r s}$ is $\prod_{j=1}^{\ell} p_{\mathrm{c}}\left(\xi_{j}\right) F_{j}\left(\xi_{j}\right)^{-1}$. Also, the formula $\omega_{h}=\sum_{\xi} p_{\text {nc }}(\xi) \omega_{\xi}$ for the Kähler quotient gives $Q=\prod_{\xi} p_{\text {nc }}(\xi)^{m_{\xi}}=$ $\pm \prod_{j=1}^{\ell} p_{\mathrm{c}}\left(\xi_{j}\right)$, cf. (48). It follows immediately from Proposition 7 that if $\kappa_{\xi}$ is a Ricci potential for $\left(S_{\xi}, \omega_{\xi}\right)$, then a Ricci potential for $(M, \omega)$ is

$$
\kappa=\sum_{\xi} \kappa_{\xi}-\frac{1}{2} \sum_{j=1}^{\ell} \log \left|F_{j}\left(\xi_{j}\right)\right| .
$$

In order to obtain the scalar curvature from this, we need a formula for the laplacian in the $\xi_{j}$ coordinates.

Lemma 5. For any function $f$, we have

$$
\Delta f=\Delta_{h} f-\sum_{j=1}^{\ell} \frac{1}{\Delta_{j} p_{\mathrm{c}}\left(\xi_{j}\right)} \frac{\partial}{\partial \xi_{j}}\left(F_{j}\left(\xi_{j}\right) \frac{\partial f}{\partial \xi_{j}}\right) .
$$

Proof. We just need to change coordinates in equation (39). For this observe that

$$
\begin{aligned}
& \qquad \frac{\partial}{\partial \sigma_{r}}=\sum_{k=1}^{\ell} \frac{V_{r k}}{\Delta_{k}} \frac{\partial}{\partial \xi_{k}} \quad \text { and } \quad \frac{\partial}{\partial u_{r}}=\sum_{s=1}^{\ell} H_{r s} \frac{\partial}{\partial \sigma_{s}}=\sum_{j=1}^{\ell} \frac{W_{j r} F_{j}\left(\xi_{j}\right)}{p_{\mathrm{c}}\left(\xi_{j}\right)} \frac{\partial}{\partial \xi_{j}}, \\
& \text { so that } \quad \Delta f=\Delta_{h} f-\frac{1}{Q} \sum_{r, j, k=1}^{\ell} \frac{V_{r k}}{\Delta_{k}} \frac{\partial}{\partial \xi_{k}}\left(\frac{Q W_{j r} F_{j}\left(\xi_{j}\right)}{p_{\mathrm{c}}\left(\xi_{j}\right)} \frac{\partial f}{\partial \xi_{j}}\right) .
\end{aligned}
$$

Since $Q= \pm \prod_{i=1}^{\ell} p_{\mathrm{c}}\left(\xi_{i}\right)$, this agrees with (78) once we observe that

$$
\begin{aligned}
\sum_{k, r=1}^{\ell} \frac{V_{r k}}{\Delta_{k}} \frac{\partial W_{j r}}{\partial \xi_{k}} & =-\sum_{r, k=1}^{\ell} \frac{W_{j r}}{\Delta_{k}} \frac{\partial V_{r k}}{\partial \xi_{k}} \\
& =\sum_{r=1}^{\ell-1} \sum_{k=1}^{\ell} \frac{W_{j r}}{\Delta_{k}}(-1)^{r}(\ell-r) \xi_{k}^{\ell-r-1}=0
\end{aligned}
$$

by the Vandermonde identity. 
Applying this formula to the Ricci potential, we deduce immediately that

$$
\text { Scal }=\sum_{\xi} \frac{\operatorname{Scal}_{g_{\xi}}}{p_{\mathrm{nc}}(\xi)}-\sum_{j=1}^{\ell} \frac{F_{j}^{\prime \prime}\left(\xi_{j}\right)}{\Delta_{j} p_{\mathrm{c}}\left(\xi_{j}\right)},
$$

where $\mathrm{Scal}_{g_{\xi}}$ is the scalar curvature of the (possibly negative definite) metric $g_{\xi}$.

Lemma 6. Suppose that Scal depends polynomially on $\xi_{1}, \ldots, \xi_{\ell}$ $(\ell>0)$. Then

- for all $j, F_{j}^{\prime \prime}(t)=\check{p_{\mathrm{c}}}(t) R(t)$, where $\check{p_{\mathrm{c}}}(t)=\prod_{\xi}(t-\xi)^{m_{\xi}-1}$ and $R(t)$ is a polynomial independent of $j$;

- for all $\xi,\left(g_{\xi}, \omega_{\xi}\right)$ has $\operatorname{Scal}_{g_{\xi}}=-R(\xi) / \prod_{\eta \neq \xi}(\xi-\eta)$.

We then have

$$
\begin{aligned}
\text { Scal }= & -\sum_{\xi} \frac{R(\xi)}{\prod_{\eta \neq \xi}(\xi-\eta) \prod_{k}\left(\xi-\xi_{k}\right)} \\
& -\sum_{j=1}^{\ell} \frac{R\left(\xi_{j}\right)}{\prod_{k \neq j}\left(\xi_{j}-\xi_{k}\right) \prod_{\eta}\left(\xi_{j}-\eta\right)},
\end{aligned}
$$

where $\check{m}=\ell+\sum_{\xi} 1=m-\sum_{\xi}\left(m_{\xi}-1\right)$.

Furthermore, if Scal has degree $\leq q$ in each variable $\xi_{j}$, then $R(t)$ has degree at most $\check{m}+q-1$. Hence, for each $j, F_{j}(t)$ is a polynomial of degree at most $m+q+1$.

Proof. We multiply the formula (79) by $\Delta_{k} p_{\mathrm{c}}\left(\xi_{k}\right)$ to obtain an equality between polynomials in $\xi_{k}$ (in a nonempty open set, hence everywhere):

$$
\begin{aligned}
& \Delta_{k} p_{\mathrm{c}}\left(\xi_{k}\right) \text { Scal } \\
& =-\sum_{\xi} \frac{\Delta_{k} \check{p_{\mathrm{c}}}\left(\xi_{k}\right) \prod_{\eta \neq \xi}\left(\xi_{k}-\eta\right)}{\prod_{j \neq k}\left(\xi-\xi_{j}\right)} \operatorname{Scal}_{g_{\xi}}-F_{k}^{\prime \prime}\left(\xi_{k}\right)-\sum_{j \neq k} \frac{\Delta_{k} p_{\mathrm{c}}\left(\xi_{k}\right)}{\Delta_{j} p_{\mathrm{c}}\left(\xi_{j}\right)} F_{j}^{\prime \prime}\left(\xi_{j}\right) .
\end{aligned}
$$

This clearly shows that $F_{k}^{\prime \prime}$ is a polynomial with $\check{p_{\mathrm{c}}}$ as a factor. Evaluating at $\xi_{k}=\xi_{j}$ for some fixed $j$, we obtain $F_{k}^{\prime \prime}\left(\xi_{j}\right)=F_{j}^{\prime \prime}\left(\xi_{j}\right)$ for all $\xi_{j}$ (in a nonempty open set, hence everywhere). Dividing through by $\check{p_{\mathrm{c}}}\left(\xi_{k}\right)$ we now have

$$
\begin{aligned}
& \Delta_{k} \prod_{\eta}\left(\xi_{k}-\eta\right) \mathrm{Scal} \\
& =-\sum_{\xi} \frac{\Delta_{k} \prod_{\eta \neq \xi}\left(\xi_{k}-\eta\right)}{\prod_{j \neq k}\left(\xi-\xi_{j}\right)} \operatorname{Scal}_{g_{\xi}}-R\left(\xi_{k}\right)-\sum_{j \neq k} \frac{\Delta_{k} \prod_{\eta}\left(\xi_{k}-\eta\right)}{\Delta_{j} \prod_{\eta}\left(\xi_{j}-\eta\right)} R\left(\xi_{j}\right) .
\end{aligned}
$$


Evaluating at $\xi_{k}=\xi$ gives the formula for $\mathrm{Scal}_{g_{\xi}}$, and it is straightforward to count the degree in $\xi_{k}$. Dividing by $\Delta_{k} \prod_{\eta}\left(\xi_{k}-\eta\right)$ now gives (80).

q.e.d.

To interpret (80), we adjoin the distinct constant roots to the variables $\xi_{1}, \ldots \xi_{\ell}$. If we label these $\xi_{1}, \ldots \xi_{\ell}, \xi_{\ell+1}, \ldots \xi_{\check{m}}$ and let $\Delta_{j}^{\vee}=$ $\prod_{k \neq j}\left(\xi_{j}-\xi_{k}\right)$, where the product is over $k=1, \ldots \check{m}$, then the right hand side of (80) is just $-\sum_{j=1}^{\check{m}} R\left(\xi_{j}\right) / \Delta_{j}^{\vee}$ which is a polynomial of degree at most $q$ in each $\xi_{j}$, by the Vandermonde identity.

5.2. Extremal Kähler metrics. Recall that a Kähler metric is called extremal if the scalar curvature is a Killing potential [10]. Weakly Bochner-flat Kähler metrics of dimension $2 m \geq 4$ are extremal, since the scalar curvature is then the trace of a hamiltonian 2-form. In this section we classify the extremal Kähler metrics with a hamiltonian 2-form such that $d_{h}$ Scal $=0$.

Proposition 15. Let $(M, g, J, \omega)$ be Kähler with a hamiltonian 2form of order $\ell>0$. Then Scal is a hamiltonian for a Killing vector field tangent to the fibres of $M$ over the Kähler quotient $S$ if and only if $(g, J, \omega)$ has the form (63) where:

- for all $j, F_{j}^{\prime \prime}(t)=\check{p_{\mathrm{c}}}(t)\left(\sum_{r=0}^{\check{m}} a_{r} t^{\check{m}-r}\right)$, and $a_{0}, \ldots a_{\check{m}}$ are arbitrary constants (independent of $j$ );

- for all $\xi,\left(g_{\xi}, \omega_{\xi}\right)$ has $\operatorname{Scal}_{g_{\xi}}=-\left(\sum_{r=0}^{\check{m}} a_{r} \xi^{\check{m}-r}\right) / \prod_{\eta \neq \xi}(\xi-\eta)$.

The scalar curvature of $(g, J, \omega)$ is then given by Scal $=-\left(a_{0} \check{\sigma}_{1}+a_{1}\right)$, where $\check{\sigma}_{1}:=\sum_{j=1}^{\check{m}} \xi_{j}=\sigma_{1}+\sum_{\xi} \xi$, so that Scal is a hamiltonian for $-a_{0} K_{1}$.

Any constant scalar curvature Kähler metric with a hamiltonian 2form arises in this way with $a_{0}=0$, and is scalar-flat if and only if $a_{1}=0$.

Proof. Since Scal is invariant under $K_{1}, \ldots K_{\ell}$, it must be a function of $\sigma_{1}, \ldots \sigma_{\ell}$, and since $J \operatorname{grad}_{g}$ Scal commutes with $K_{1}, \ldots K_{\ell}$ and is in their span at each point, it must in fact be a constant linear combination of $K_{1}, \ldots K_{\ell}$, so that Scal is an affine function of $\sigma_{1}, \ldots \sigma_{\ell}$. Now any such function is a polynomial in $\xi_{1}, \ldots \xi_{\ell}$ of degree one in each $\xi_{j}$. Hence we can apply Lemma 6.

Conversely, the Vandermonde identities imply that Scal $=-\left(a_{0} \check{\sigma}_{1}+\right.$ $a_{1}$ ), which is a Killing potential for $-a_{0} K_{1}$, since $\check{\sigma}_{1}$ differs from $\sigma_{1}$ by a constant.

q.e.d.

5.3. Weakly Bochner-flat Kähler metrics. On a weakly Bochnerflat Kähler manifold of dimension $2 m \geq 4, \tilde{\rho}$ is a hamiltonian 2-form, so we obtain a classification by specializing the work of the previous section to the case $\tilde{\rho}=\phi$. In fact we may as well consider more generally the case that $\tilde{\rho}=a \phi+b \omega$ for constants $a, b$. Then when $a=0$, we will 
have characterized the Kähler-Einstein metrics among Kähler metrics with a hamiltonian 2-form. Note, however, that we have fixed $\phi$ : it is obviously possible for $\tilde{\rho}$ to be a hamiltonian 2 -form without it being equal to $a \phi+b \omega$ for a given $\phi$, but we have nothing to say about this situation.

Proposition 16. Let $(M, g, J, \omega)$ be Kähler of dimension $2 m \geq 4$ with a hamiltonian 2-form $\phi$. Then $M$ is weakly Bochner-flat with $\tilde{\rho}$ a linear combination of $\phi$ and $\omega$ if and only if $(g, J, \omega)$ has the explicit form (63) where:

- for all $j, F_{j}^{\prime}(t)=p_{\mathrm{c}}(t)\left(\sum_{r=-1}^{\ell} b_{r} t^{\ell-r}\right)$, and $b_{-1}, \ldots b_{\ell}$ are arbitrary constants (independent of $j$ );

- for all $\xi,\left(g_{\xi}, \omega_{\xi}\right)$ is Kähler-Einstein with Kähler-Einstein constant

$$
\frac{1}{m_{\xi}} \operatorname{Scal}_{g_{\xi}}=-\sum_{r=-1}^{\ell} b_{r} \xi^{\ell-r} .
$$

The Ricci form of $(g, J, \omega)$ is then given by $\rho=-\frac{1}{2}\left(b_{-1}\left(\phi+\sigma_{1} \omega\right)+b_{0} \omega\right)$.

Any Kähler-Einstein metric with a hamiltonian 2-form arises in this way with $b_{-1}=0$, and is Ricci-flat if and only if $b_{0}=0$.

Proof. $-2 \rho=b_{-1}\left(\phi+\sigma_{1} \omega\right)+b_{0} \omega$ if and only if $d d^{c}$ potentials for the two sides differ by a pluriharmonic function. This means that $-2 \kappa$ must be of the form $-\sum_{r=-1}^{\ell}(-1)^{r} b_{r} u_{r}$ where

$$
u_{r}=-H_{r}-\sum_{j=1}^{\ell} \int^{\xi_{j}} \frac{(-1)^{r} t^{\ell-r} p_{\mathrm{c}}(t)}{F_{j}(t)} d t
$$

for $r=-1, \ldots \ell$, the $H_{r}$ being functions on $\prod_{\xi} S_{\xi}$ such that $H_{r}$ is a $d d^{c}$-potential for $\Omega_{r}=\sum_{\xi}(-1)^{r} \xi^{\ell-r} \omega_{\xi}$ when $r=0, \ldots \ell$. (So $u_{-1}$ is a $d d^{c}$ potential for $\phi+\sigma_{1} \omega, u_{0}$ is a $d d^{c}$ potential for $-\omega$ and $u_{r}$ is pluriharmonic for $r \geq 1$ - see Remark 5.) Now it follows from (77) that

$$
-2 \kappa=-2 \sum_{\xi} \kappa_{\xi}+\sum_{j=1}^{\ell} \int^{\xi_{j}} \frac{F_{j}^{\prime}(t)}{F_{j}(t)} d t .
$$

Comparing this with

$$
\sum_{r=-1}^{\ell}(-1)^{r} b_{r} H_{r}+\sum_{j=1}^{\ell} \int^{\xi_{j}} \frac{p_{\mathrm{c}}(t)\left(\sum_{r=-1}^{\ell} b_{r} t^{\ell-r}\right)}{F_{j}(t)} d t
$$

we obtain the required result.

q.e.d. 
5.4. Bochner-flat Kähler metrics. We now rederive Bryant's classification of Bochner-flat Kähler metrics in the present framework. One interesting feature of our approach is that we can at the same time explicitly classify hamiltonian 2-forms on Bochner-flat Kähler-Einstein manifolds, i.e., on Kähler manifolds of constant holomorphic sectional curvature, cf. section 2.4.

We set $\hat{p}_{\mathrm{c}}(t)=\prod_{\xi}(t-\xi)^{m_{\xi}+1}$ and $\hat{m}=\ell-\sum_{\xi} 1=m-\sum_{\xi}\left(m_{\xi}+1\right)$.

Proposition $17([\mathbf{8}])$. Let $(M, g, J, \omega)$ be a Kähler manifold of dimension $2 m \geq 4$ with a hamiltonian 2-form. Then $M$ is Bochner-flat with $\tilde{\rho}$ a linear combination of $\phi$ and $\omega$ if and only if $(g, J, \omega)$ has the explicit form (63) where:

- for all $j, F_{j}(t)=\hat{p}_{\mathrm{c}}(t)\left(\sum_{r=-2}^{\hat{m}} c_{r} t^{\hat{m}-r}\right)$, and $c_{-2}, \ldots c_{\hat{m}}$ are arbitrary constants (independent of $j$ );

- for all $\xi,\left(g_{\xi}, \omega_{\xi}\right)$ has constant holomorphic sectional curvature

$$
\frac{1}{m_{\xi}\left(m_{\xi}+1\right)} \operatorname{Scal}_{g_{\xi}}=-\left(\sum_{r=-2}^{\hat{m}} c_{r} \xi^{\hat{m}-r}\right) \prod_{\eta \neq \xi}(\xi-\eta) .
$$

The curvature of $(g, J, \omega)$ is then given by $R=-\{J \circ \hat{\rho}, \cdot\}+\hat{\rho} \otimes \omega+\omega \otimes \hat{\rho}$, where $\hat{\rho}=-\frac{1}{2}\left(c_{-2}\left(\phi+\frac{1}{2} \hat{\sigma}_{1} \omega\right)+\frac{1}{2} c_{-1} \omega\right)$ and $\hat{\sigma}_{1}=\sigma_{1}-\sum_{\xi} \xi$.

Any constant holomorphic sectional curvature Kähler metric with a hamiltonian 2-form arises in this way with $c_{-2}=0$, and is flat if and only if $c_{-1}=0$.

Proof. By Proposition 16, we may assume that $(g, J, \omega)$ is weakly Bochner-flat, with $F_{j}^{\prime}$ a polynomial divisible by $p_{\mathrm{c}}$ and $S_{\xi}$ KählerEinstein. Since $\tilde{\rho}$ is a linear combination of $\phi$ and $\omega$, we may use the system (30). Integrating the last three equations gives $\tau_{0}=C_{-2}$, $\tau_{1}=C_{-2} \sigma_{1}+C_{-1}$ and $\tau_{2}=C_{-2}\left(\sigma_{1}^{2}-\sigma_{2}\right)+C_{-1} \sigma_{1}+C_{0}$ for constants $C_{-2}$, $C_{-1}$ and $C_{0}$. (We have used the fact that $\sigma-\sigma_{1}$ and $\langle\phi, \phi\rangle+2 \sigma_{2}-\sigma_{1}^{2}$ are constants.) Hence

$$
\begin{aligned}
d d^{c} \sigma_{1}= & \frac{2}{m} W^{\mathcal{K}}(\phi)-C_{-2} J \phi^{2}+\left(C_{-2} \sigma_{1}+C_{-1}\right) \phi \\
& +\left(C_{-2}\left(\sigma_{1}^{2}-\sigma_{2}\right)+C_{-1} \sigma_{1}+C_{0}\right) \omega \\
= & \frac{2}{m} W^{\mathcal{K}}(\phi)-C_{-2}\left(J \phi^{2}-\sigma_{1} \phi-\left(\sigma_{1}^{2}-\sigma_{2}\right) \omega\right) \\
& +C_{-1}\left(\phi+\sigma_{1} \omega\right)+C_{0} \omega .
\end{aligned}
$$

It follows from Remark 5 that

$$
\frac{2}{m} W^{\mathcal{K}}(\phi)=d d^{c}\left(\sigma_{1}+C_{-2} u_{-2}-C_{-1} u_{-1}+C_{0} u_{0}\right) .
$$

(Here we recall that $d d^{c} u_{-2}=J \phi^{2}-\sigma_{1} \phi-\left(\sigma_{1}^{2}-\sigma_{2}\right) \omega$.) 
Thus $W^{\mathcal{K}}(\phi)$ is basic if and only if $F_{j}(t)=p_{\mathrm{c}}(t)\left(\sum_{r=-2}^{\ell} C_{r} t^{\ell-r}\right)$, where $C_{1}, \ldots C_{\ell}$ are arbitrary constants. This means that $F_{j}$ is a polynomial divisible by $p_{\mathrm{c}}$. Since $F_{j}^{\prime}$ is also divisible by $p_{\mathrm{c}}, W^{\mathcal{K}}(\phi)$ is basic if and only if $F_{j}$ is a polynomial divisible by $\hat{p}_{\mathrm{c}}$. In particular $\sum_{r=-2}^{\ell} C_{r} \xi^{\ell-r}=0$ for each constant root $\xi$, and so

$$
\begin{aligned}
\frac{2}{m} W^{\mathcal{K}}(\phi) & =d d^{c}\left(\sigma_{1}+\sum_{r=-2}^{\ell}(-1)^{r} C_{r} u_{r}\right) \\
& =-\sum_{r=-2}^{\ell}(-1)^{r} C_{r} d d^{c} H_{r}=\sum_{r=-2}^{\ell} \sum_{\xi} C_{r} \xi^{\ell-r} \omega_{\xi}=0 .
\end{aligned}
$$

Hence we may suppose that $F_{j}(t)=\hat{p}_{\mathrm{c}}(t)\left(\sum_{r=-2}^{\hat{m}} c_{r} t^{\hat{m}-r}\right)$ and that $W^{\mathcal{K}}(\phi)=0$. Note that $c_{-2}=C_{-2}$ and $c_{-1}=C_{-1}+\left(\sum_{\xi} \xi\right) C_{-2}$, so $\tau_{0}=c_{-2}$ and $\tau_{1}=c_{-2} \hat{\sigma}_{1}+c_{-1}$.

Since $\tilde{\rho}$ is an constant linear combination of $\phi$ and $\omega$, equation (28) implies that $\left[W^{\mathcal{K}}(\psi), \phi\right]=\frac{1}{m}\left[W^{\mathcal{K}}(\phi), \psi\right]=0$. Also equation (29) implies that $\iota_{K} W^{\mathcal{K}}(\psi)=0$. Now any 2 -form commuting with $\phi$ is the sum of a vertical and a horizontal 2 -form (i.e., there is no mixed component); then since $-J \phi$ has distinct eigenvalues on the fibres of $M$ over $S$, the vertical component is of the form $\sum_{j=1}^{\ell} \mu_{j} d \xi_{j} \wedge J d \xi_{j}$. If also the contraction with $K$ is zero, we have $\sum_{j=1}^{\ell} \mu_{j} d \xi_{j}(X)\left|d \xi_{j}\right|^{2}=0$, for each vector field $X$, which forces $\mu_{1}=\cdots=\mu_{r}=0$. We deduce that for any 2-form $\psi, W^{\mathcal{K}}(\psi)$ is horizontal, and, since $W^{\mathcal{K}}$ is symmetric, $W^{\mathcal{K}}(\psi)$ vanishes unless $\psi$ is horizontal.

We next employ the Gray-O'Neill submersion formulae $[\mathbf{1 3}, \mathbf{2 1}]$ which apply in this situation (the submersion of $M$ over $S$ is not riemannian, so we need the framework of Gray). If $X, Y, Z$ are horizontal vector fields, we have (see Proposition 9)

$$
\begin{aligned}
\left(R_{X, Y} Z\right)^{H}=R_{X, Y}^{H} Z & -\langle C(X), C(Y, Z)\rangle^{\sharp}+\langle C(Y), C(X, Z)\rangle^{\sharp} \\
& +\langle C(Z), C(X, Y)\rangle^{\sharp}-\langle C(Z), C(Y, X)\rangle^{\sharp},
\end{aligned}
$$

with $2 C(X, Y)=\sum_{r=1}^{\ell}\left(\Omega_{r}(X, Y) K_{r}+\Omega_{r}(J X, Y) J K_{r}\right)$.

To compute $\langle C(X, \tilde{X}), C(Y, \tilde{Y})\rangle$ for horizontal vector fields $X, \tilde{X}, Y, \tilde{Y}$, we use the definition of $C$, and expand $\Omega_{r}$ and $\left\langle K_{r}, K_{s}\right\rangle$ to get

$$
\begin{aligned}
& \langle C(X, \tilde{X}), C(Y, \tilde{Y})\rangle \\
& =\frac{1}{4} \sum_{\xi, \eta} f_{\xi, \eta}\left(\omega_{\xi}(X, \tilde{X}) \omega_{\eta}(Y, \tilde{Y})+\omega_{\xi}(J X, \tilde{X}) \omega_{\eta}(J Y, \tilde{Y})\right),
\end{aligned}
$$


where

$$
\begin{aligned}
f_{\xi, \eta}= & \sum_{j=1}^{\ell} \frac{F_{j}\left(\xi_{j}\right)}{p_{\mathrm{c}}\left(\xi_{j}\right) \Delta_{j}}\left(\sum_{r=1}^{\ell}(-1)^{r} \xi^{\ell-r} \sigma_{r-1}\left(\hat{\xi}_{j}\right)\right)\left(\sum_{s=1}^{\ell}(-1)^{s} \eta^{\ell-s} \sigma_{s-1}\left(\hat{\xi}_{j}\right)\right) \\
= & \left(\prod_{k=1}^{\ell}\left(\xi-\xi_{k}\right)\left(\eta-\xi_{k}\right)\right)\left(\sum_{j=1}^{\ell} \frac{F_{j}\left(\xi_{j}\right)}{p_{\mathrm{c}}\left(\xi_{j}\right) \Delta_{j}\left(\xi_{j}-\xi\right)\left(\xi_{j}-\eta\right)}\right) \\
= & p_{\mathrm{nc}}(\xi) p_{\mathrm{nc}}(\eta)\left(C_{-2}\left(\sigma_{1}+\xi+\eta\right)+C_{-1}\right. \\
& \left.-\delta_{\xi, \eta} \frac{\left(\sum_{r=-2}^{\hat{m}} c_{r} \xi^{\hat{m}-r}\right) \prod_{\eta \neq \xi}(\xi-\eta)}{\prod_{j=1}^{\ell}\left(\xi-\xi_{j}\right)}\right) .
\end{aligned}
$$

For the last line of this calculation, we observe that $F_{j}(t) / p_{\mathrm{c}}(t)$ is a polynomial of degree $\ell+2$ vanishing when $t=\xi$; then if $\xi \neq \eta$ we can apply the Vandermonde identity with the variables $\xi_{1}, \ldots \xi_{\ell}, \xi, \eta$, whereas if $\xi=\eta$, we may use the Vandermonde identity for the polynomial $F_{j}(t) /\left(p_{\mathrm{c}}(t)(t-\xi)\right)=\left(\sum_{r=-2}^{\hat{m}} c_{r} t^{\hat{m}-r}\right) \prod_{\eta \neq \xi}(t-\eta)$ of degree $\ell+1$, with the variables $\xi_{1}, \ldots \xi_{\ell}, \xi$.

We expand the curvature $R$ using (26) and the fact that

$$
\begin{aligned}
\hat{\rho}=-\frac{1}{2}\left(\tau_{0} \phi+\frac{1}{2} \tau_{1} \omega\right) & =-\frac{1}{2} C_{-2}\left(\phi+\frac{1}{2} \sigma_{1} \omega\right)-\frac{1}{4} C_{-1} \omega \\
& =-\frac{1}{2} c_{-2}\left(\phi+\frac{1}{2} \hat{\sigma}_{1} \omega\right)-\frac{1}{4} c_{-1} \omega .
\end{aligned}
$$

(See section 2.3.) The final ingredient in the computation, from Proposition 16, is the fact that each $S_{\xi}$ is Kähler-Einstein with Kähler-Einstein constant

$$
\frac{1}{m_{\xi}} \operatorname{Scal}_{g_{\xi}}=-\left(m_{\xi}+1\right)\left(\sum_{r=-2}^{\hat{m}} c_{r} \xi^{\hat{m}-r}\right) \prod_{\eta \neq \xi}(\xi-\eta) .
$$

Putting these ingredients together, bearing in mind that $\left.\omega\right|_{S_{\xi}}=$ $p_{\text {nc }}(\xi) \omega_{\xi}$, we find that a remarkable cancellation occurs (cf. $\left.[8]\right)$ and we obtain

$$
\left(W_{X, Y}^{\mathcal{K}} Z\right)^{H}=\sum_{\xi} W_{X, Y}^{\mathcal{K}, S_{\xi}} Z
$$

where $W^{\mathcal{K}, S_{\xi}}$ denotes the Bochner tensor of $S_{\xi}$ (pulled back to the Kähler product). We deduce that $\left(W_{X, Y}^{\mathcal{K}} Z\right)^{H}=0$ if and only if each $S_{\xi}$ has constant holomorphic sectional curvature given by the stated formula.

q.e.d.

Our proof above is very much inspired by [8, Section 4.5], where Bryant indicates how to obtain an explicit formula for the general Bochner-flat metric, although he stops short of providing the final formula. Our approach has proceeded in reverse, by first finding the general formula, then showing that it is Bochner-flat. This has permitted us to 
give a proof using standard methods in Kähler geometry, substituting a linear system for the nonlinear system which Bryant integrates using Cartan's generalization of Lie's Third Theorem.

Bryant's remarkable paper also addresses global questions: the compact Bochner-flat Kähler manifolds are necessarily locally symmetric, but Bryant finds compact orbifold examples, and classifies the complete examples (cf. also [2] for the case of Kähler surfaces). In subsequent work $[\mathbf{4}, \mathbf{6}]$, we find that there are many compact weakly Bochner-flat Kähler manifolds (see also $[\mathbf{3}]$ ).

We now return to the characteristic polynomial of section 2.3. We write $F$ for $F_{j}$ (which is independent of $j$ ), and define a minimal polynomial $F_{\mathrm{m}}(t):=F(t) / p_{\mathrm{c}}(t)=\left(\sum_{r=-2}^{\hat{m}} c_{r} t^{\hat{m}-r}\right) \prod_{\xi}(t-\xi)$. When $c_{-2} \neq 0$, these polynomials are (up to affine transformation of $t$ ) Bryant's characteristic and reduced characteristic polynomials.

Proposition 18. Let $\phi$ be a hamiltonian 2-form on a Bochner-flat Kähler manifold $(M, g, J, \omega)$ with $\tilde{\rho}$ a linear combination of $\phi$ and $\omega$, as in Proposition 17. Then $F$ is the characteristic polynomial $F_{\mathrm{c}}$ of $(g, J, \omega, \phi)$.

Proof. To compute $\left(\tau_{0} t^{2}+\tau_{1} t+\tau_{2}\right) p(t)-\langle K, K(t)\rangle$, observe that

$$
\begin{aligned}
\langle K, K(t)\rangle & =-\sum_{j=1}^{\ell} p_{\mathrm{c}}(t)\left(\prod_{k \neq j}\left(t-\xi_{j}\right)\right)\left|d \xi_{j}\right|^{2} \\
& =p(t) \sum_{j=1}^{\ell} \frac{F_{\mathrm{m}}\left(\xi_{j}\right)}{\left(\xi_{j}-t\right) \Delta_{j}} .
\end{aligned}
$$

Hence, writing $F_{\mathrm{m}}(t)=\sum_{r=-2}^{\ell} C_{r} t^{\ell-r}$, Vandermonde identities (with variables $\left.\xi_{1}, \ldots \xi_{\ell}, t\right)$ give

$$
\begin{aligned}
& \langle K, K(t)\rangle \\
& =p(t)\left(C_{-2}\left(\left(\sigma_{1}+t\right)^{2}-\sigma_{2}-t \sigma_{1}\right)+C_{-1}\left(\sigma_{1}+t\right)+C_{0}\right)-p(t) \frac{F_{\mathrm{m}}(t)}{p_{\mathrm{nc}}(t)} \\
& =p(t)\left(C_{-2} t^{2}+\left(C_{-2} \sigma_{1}+C_{-1}\right) t\right. \\
& \quad+\left(C_{-2}\left(\sigma_{1}^{2}-\sigma_{2}\right)+C_{-1} \sigma_{1}+C_{0}\right)-F(t) .
\end{aligned}
$$

Hence $\left(\tau_{0} t^{2}+\tau_{1} t+\tau_{2}\right) p(t)-\langle K, K(t)\rangle=F(t)$.

q.e.d.

When $c_{-2}=0$ (i.e., $\tau_{0}=0$ ), Proposition 17 provides a classification of hamiltonian 2-forms on simply-connected manifolds of constant holomorphic sectional curvature in terms of two polynomials $p_{\mathrm{c}}$ and $F_{\mathrm{m}}$ respectively of degrees $m-\ell$ (precisely) and $\ell+1$ (at most), such that every root of $p_{\mathrm{c}}$ is a root of $F_{\mathrm{m}}$.

In section 2.4 we showed that hamiltonian 2 -forms are then given by parallel sections of a flat connection on a bundle of rank $(m+1)^{2}$. In the 
simply-connected case, or when the Kähler manifold is an open subset of $\mathbb{C}^{m}, \mathbb{C} P^{m}$ or $\mathbb{C} \mathcal{H}^{m}$, the bundle is trivial, and parallel sections extend globally to $\mathbb{C}^{m}, \mathbb{C} P^{m}$ or $\mathbb{C} \mathcal{H}^{m}$. For $s \neq 0$, we identified the solution space with the Lie algebra $\mathfrak{u}(m+1)$ or $\mathfrak{u}(m, 1)$ of Killing potentials, and we gave an explicit description for $s=0$.

The positive-definiteness of the explicit metric $g$ implies that $F_{\mathrm{m}}\left(\xi_{j}\right) / \Delta_{j}$ must be positive for all $j$, so $F_{\mathrm{m}}$ must have at least $\ell-1$ distinct roots (without loss of generality $\xi_{1}<\cdots<\xi_{\ell}$ and $F_{\mathrm{m}}$ has a sign change in each interval). The scalar curvature is nonzero when $F_{\mathrm{m}}$ has exactly degree $\ell+1$. In the positive case $\left(C_{-1}<0\right)$, it changes sign in $\left(-\infty, \xi_{1}\right)$ and $\left(\xi_{\ell}, \infty\right)$ and so has $\ell+1$ distinct roots; the roots of $p_{\mathrm{c}}$ are thus the multiple roots of the characteristic polynomial $F$, and the order $\ell$ of $\phi$ equals the number of different roots of the characteristic polynomial minus one. (The case of only one root is the trivial case that $\tau$ is constant and $\phi=\tau \omega$.) The negative case is more complicated, corresponding to the fact that $\mathfrak{u}(m, 1)$ has non-semisimple elements, and $F$ alone is not enough to classify them-we also need to know the factorization $F(t)=F_{\mathrm{m}}(t) p_{\mathrm{c}}(t)$, i.e., the minimal polynomial.

5.5. The Calabi-type case. To illustrate the conditions of the previous subsections, and for use in the next subsection, we specialize to the simple but important case of hamiltonian 2-forms of order one, when the Kähler structure may be written:

$$
\begin{array}{rlrl}
g & =\sum_{\xi}(z-\xi) g_{\xi}+\frac{\prod_{\xi}(z-\xi)^{m_{\xi}}}{F(z)} d z^{2}+\frac{F(z)}{\prod_{\xi}(z-\xi)^{m_{\xi}}} \theta^{2}, \\
\omega & =\sum_{\xi}(z-\xi) \omega_{\xi}+d z \wedge \theta, & d \theta & =\sum_{\xi} \omega_{\xi}, \\
J d z & =\frac{F(z)}{\prod_{\xi}(z-\xi)^{m_{\xi}}} \theta, & J \theta & =-\frac{\prod_{\xi}(z-\xi)^{m_{\xi}}}{F(z)} d z,
\end{array}
$$

where $2 m_{\xi}=\operatorname{dim} S_{\xi}$ and $\pm\left(g_{\xi}, \omega_{\xi}\right)$ is a Kähler metric on $S_{\xi}$ (compared to $(63)$, and we have reversed the sign of $\left.\left(g_{\xi}, \omega_{\xi}\right)\right)$. Suppose there are $N$ different constant roots $\xi$. Then Propositions 15, 16 and 17 specialize as follows:

(i) $g$ is extremal when $P_{2}(t):=F^{\prime \prime}(t) / \prod_{\xi}(t-\xi)^{m_{\xi}-1}$ is a polynomial of degree $N+1$ and $g_{\xi}$ has constant scalar curvature $P_{2}(\xi) / \prod_{\eta \neq \xi}(\xi-\eta)$;

$g$ has constant scalar curvature when $P_{2}$ has degree $N$;

(ii) $g$ is weakly Bochner-flat when $P_{1}(t):=F^{\prime}(t) / \prod_{\xi}(t-\xi)^{m_{\xi}}$ is a polynomial of degree 2 and $g_{\xi}$ is Kähler-Einstein with Kähler-Einstein constant $P_{1}(\xi)$;

$g$ is Kähler-Einstein when $P_{1}$ has degree 1;

(iii) $g$ is Bochner-flat when $P_{0}(t):=F(t) / \prod_{\xi}(t-\xi)^{m_{\xi}+1}$ is a polynomial of degree $3-N$ and $S_{\xi}$ has constant holomorphic sectional curvature $P_{0}(\xi) \prod_{\eta \neq \xi}(\eta-\xi)$ 
$g$ has constant holomorphic sectional curvature when $P_{0}$ has degree $2-N$.

Metrics of this form have been used in many places to provide compact or complete examples on (projective) line bundles over Kähler products: see $[\mathbf{1 7}, \mathbf{2 7}]$ for Kähler-Einstein metrics, and $[\mathbf{1 0}, \mathbf{1 5}, \mathbf{2 5}, \mathbf{2 6}]$ for extremal Kähler metrics. We shall explore and generalize these global examples in subsequent work $[\mathbf{4}, \mathbf{5}, \mathbf{6}]$.

The case $N=1$ is particularly important: the constant roots are all equal, so without loss of generality we may take them all to be zero. The polynomials $P_{0}, P_{1}, P_{2}$ are all quadratic: in case (i), we may write $F(t)=c_{-2} t^{m+2}+c_{-1} t^{m+1}+c_{0} t^{m}+b t+a$ and the scalar curvature of $g_{0}$ is $m(m-1) c_{0}$; then case (ii) has $b=0$ and Kähler-Einstein constant $m c_{0}$, while case (iii) has $a=b=0$ with holomorphic sectional curvature $c_{0}$. The Kähler structure is

$$
\begin{aligned}
g & =z g_{0}+\frac{z^{m-1}}{F(z)} d z^{2}+\frac{F(z)}{z^{m-1}} \theta^{2}, & \omega & =z \omega_{0}+d z \wedge \theta, \\
J d z & =\frac{F(z)}{z^{m-1}} \theta, \quad J \theta=-\frac{z^{m-1}}{F(z)} d z, & d \theta & =\omega_{0},
\end{aligned}
$$

which is the local form of a Kähler metric of Calabi's type [9] on a line bundle over a Kähler manifold $\left(S, g_{0}, \omega_{0}\right)$ of dimension $2(m-1)$ Calabi's extremal Kähler metrics [10] were constructed in this way.

We remark that any such metric (81) is conformal to a Kähler metric of the same form, but with an oppositely oriented complex structure. Indeed we may set $\tilde{z}=1 / z, \tilde{g}=g / z^{2}$ and $\tilde{F}(\tilde{z}) / \tilde{z}^{m}=F(z) / z^{m}$.

5.6. Strongly conformally Einstein Kähler metrics. We return briefly to the strongly conformally Einstein manifolds of DerdzińskiMaschler [11] and explain how their classification can be derived in a natural way in our framework.

We recall that if $(M, g, J, \omega)$ is strongly conformally Einstein with conformal factor $\tau$, then $\phi=(a \tau+b) d \tau \wedge d^{c} \tau /|d \tau|^{2}$ is a hamiltonian 2 -form with trace $z=a \tau+b$. If $a=0$ then $\phi$ is parallel, and $M$ is a Kähler product of a Riemann surface and a Kähler manifold of dimension $2 m-2$. We shall concentrate on the more interesting case $a \neq 0$. We set $a=1 / q$ and $b=-p / q$ so that $\tau=q z+p$. This allows us to take the limit $q \rightarrow 0$, when $\tau$ is constant and $(M, g, J, \omega)$ is Kähler-Einstein. Since $\phi$ is hamiltonian of order one, with all constant roots zero, the Kähler structure has the explicit form (81) given in the previous subsection. This will be conformally Einstein with conformal factor $q z+p$ if and only if

$$
q d d^{c} z=\xi(z) d d^{c} \kappa+\eta(z) \omega,
$$

where $\kappa=\kappa_{0}-\frac{1}{2} \log |F(z)|, \kappa_{0}$ is a Ricci potential for $\omega_{0}, \xi(z)=$ $-(q z+p) /(m-1)$ and $\eta(z)$ is an arbitrary function. Such an equation 
can only hold if $\omega_{0}$ is Kähler Einstein, and we let $d d^{c} \kappa_{0}=c \omega_{0}$ so that Scal $_{g_{0}}=(m-1) c$. We now compute

$$
\begin{aligned}
& d d^{c} z=\frac{F}{z^{m-1}} \omega_{0}+\frac{z F^{\prime}-(m-1) F}{z^{m}} d z \wedge \theta \\
& d d^{c} \kappa=\left(c-\frac{F^{\prime}}{2 z^{m-1}}\right) \omega_{0}-\frac{z F^{\prime \prime}-(m-1) F^{\prime}}{2 z^{m}} d z \wedge \theta .
\end{aligned}
$$

Substituting in (82), we eliminate $\eta(z)$ to obtain a differential equation for $F(z)$ :

$$
\begin{aligned}
q F(z) & -\xi(z)\left(c z^{m-1}-\frac{1}{2} F^{\prime}(z)\right)=\eta(z) z^{m} \\
& =q\left(z F^{\prime}(z)-(m-1) F(z)\right)+\frac{1}{2} \xi(z)\left(z F^{\prime \prime}(z)-(m-1) F^{\prime}(z)\right) .
\end{aligned}
$$

A particular integral is $2 c z^{m} / m$ (this is the flat metric), and one solution of the homogeneous equation is $(q z-p)(q z+p)^{2 m-1}$. We can then reduce the equation to first order to find the general homogeneous solution as an integral:

$$
(q z-p)(q z+p)^{2 m-1}\left(a+b \int^{z} \frac{t^{m}}{(q t+p)^{2 m}(q t-p)^{2}} d t\right) .
$$

This is in fact a polynomial in $z$, which may be written

$$
\sum_{j=1}^{m} \frac{j}{m}\left(\begin{array}{c}
2 m \\
m+j
\end{array}\right)\left(a_{+} p^{m-j} q^{j-1} z^{m+j}-a_{-} p^{j-1} q^{m-j} z^{m-j}\right)
$$

for constants $a_{ \pm}$. The polynomial reduces to a multiple of $(q z-p)(q z+$ $p)^{2 m-1}$ when $q^{m+1} a_{-}=p^{m+1} a_{+}$, while for $a_{+} a_{-}=0$, but $p, q$ nonzero, it is a hypergeometric function of $q z / p$, essentially a Gegenbauer polynomial.

In conclusion, then, the Kähler structure (81) is conformally Einstein with conformal factor $q z+p$ if and only if

$$
F(z)=\sum_{j=1}^{m} \frac{j}{m}\left(\begin{array}{c}
2 m \\
m+j
\end{array}\right)\left(a_{+} p^{m-j} q^{j-1} z^{m+j}-a_{-} p^{j-1} q^{m-j} z^{m-j}\right)+\frac{2 c}{m} z^{m}
$$

for constants $a_{ \pm}, c$ with $\rho_{0}=c \omega_{0}$. We have

$$
\begin{aligned}
\text { Scal }= & \frac{\text { Scal }_{g_{0}}}{z}-\frac{F^{\prime \prime}(z)}{z^{m-1}} \\
= & -a_{+} \sum_{j=1}^{m} \frac{j}{m} \frac{(2 m) !}{(m+j-2) !(m-j) !} p^{m-j} q^{j-1} z^{j-1} \\
& +a_{-} \sum_{j=1}^{m-2} \frac{j}{m} \frac{(2 m) !}{(m+j) !(m-j-2) !} p^{j-1} q^{m-j} z^{-j-1},
\end{aligned}
$$


which is polynomial in $z$ if and only if $a_{-}=0, q=0$ or $m=2$. It is a Killing potential (i.e., affine in $z$ ) if and only if it vanishes or $q=0$ or $m=2$.

The function $Q(z)$ of Derdziński-Maschler is $F(z) / z^{m-1}$, and writing $\tau=q z+p$, we recover their solution, except that we have chosen the basis for the solutions in a way that unifies the cases $p=0$ and $p \neq 0$. We also recall from the previous subsection that $g$ is conformal to a Kähler metric $\tilde{g}$ with the opposite orientation. This new Kähler metric is conformally Einstein with conformal factor $\tilde{\tau}=q+p \tilde{z}$. Just as $q=0$ corresponds to the case that $g$ is Kähler-Einstein, so $p=0$ corresponds to the case that $\tilde{g}$ is Kähler-Einstein.

\section{Appendix A. Conformal Killing forms and hamiltonian 2-forms}

We have noted already that for any hamiltonian 2-form $\phi, A=\phi+\sigma \omega$ is closed. One also easily observes that $\phi-m \sigma \omega$ is co-closed (divergencefree). In this appendix we relate hamiltonian 2-forms to conformal Killing 2-forms, which have been investigated recently by Moroianu and Semmelmann [20, 24].

Definition 8. A conformal Killing or twistor 2-form on a riemannian manifold of dimension $n \geq 3$ is a 2 -form $\psi$ satisfying the equation

$$
\nabla_{X} \psi=\frac{1}{n-1} X \wedge \alpha+\frac{1}{3} \iota \iota_{X} \beta
$$

for all vector fields $X$, where $\alpha$ is a 1 -form, $\beta$ is a 3 -form and $\iota_{X}$ denotes contraction.

It follows immediately from this equation that $\alpha=-\delta \psi:=\sum_{i} \iota_{e_{i}} \nabla_{e_{i}} \psi$ and that $\beta=d \psi$. Hence $\psi$ is a conformal Killing 2 -form if and only if $\nabla \psi$, which is a section of $\Lambda^{1} M \otimes \Lambda^{2} M$, is in the image of $\Lambda^{1} M \oplus \Lambda^{3} M$ (under the natural inclusions).

The notion of conformal Killing form can be extended to $p$-forms and is conformally invariant for $p$-forms of weight 1 , i.e., for sections of $L^{p+1} \otimes \Lambda^{p} M$, where $L$ is the density line bundle, i.e., $L^{-n}=\left|\Lambda^{n} M\right|$.

The following observation is due in part to Sekizawa [23] and Semmelmann [24]. The essential new ingredient is (85).

Proposition 19. Let $(M, g, J, \omega)$ be a Kähler manifold of dimension $n=2 m \geq 4$.

(i) If $\phi$ is a hamiltonian 2-form, then the J-invariant 2-form $\psi=$ $\phi-\frac{1}{2}(\operatorname{tr} \phi) \omega$ is a conformal Killing 2 -form, with $\operatorname{tr} \psi=\left(1-\frac{m}{2}\right) \operatorname{tr} \phi$.

(ii) Conversely, if $\psi$ is a J-invariant conformal Killing 2-form, then

$$
d \psi=-\frac{3}{n-1} \omega \wedge J \delta \psi
$$


and

$$
(m-2) J \delta \psi=-(2 m-1) d \operatorname{tr} \psi
$$

Hence

$$
\nabla_{X} \psi=-\frac{1}{2 m-1}(X \wedge \delta \psi+J X \wedge J \delta \psi-\langle J \delta \psi, X\rangle \omega)
$$

and $J \delta \psi$ is closed if $m>2$, while $\operatorname{tr} \psi$ is constant if $m=2$.

(iii) If $\psi$ is a J-invariant conformal Killing 2 -form and $J \delta \psi=d f$ then $\phi=\psi+\frac{1}{2 m-1} f \omega$ is a hamiltonian 2-form.

In particular, for $m>2$ the map $\phi \mapsto \phi-\frac{1}{2}(\operatorname{tr} \phi) \omega$ is a bijection from hamiltonian 2-forms to J-invariant conformal Killing 2-forms, with inverse $\psi \mapsto \psi-\frac{1}{m-2}(\operatorname{tr} \psi) \omega$.

Proof. (i) If $\phi$ is hamiltonian and $\psi=\phi-\frac{1}{2} \sigma \omega$, then

$\nabla_{X} \psi=\frac{1}{2}\left(d \sigma \wedge J X-d^{c} \sigma \wedge X-d \sigma(X) \omega\right)=\frac{1}{2}\left(X \wedge d^{c} \sigma-\iota_{X}(\omega \wedge d \sigma)\right)$.

Hence $\psi$ is a conformal Killing 2-form with $\delta \psi=-\frac{n-1}{2} d^{c} \sigma$ and $d \psi=$ $-\frac{3}{2} \omega \wedge d \sigma$.

(ii) Observe that $\nabla_{X} \psi$ is $J$-invariant and so

$$
\begin{aligned}
& \frac{1}{3}(d \psi(X, J Y, Z)+d \psi(X, Y, J Z)) \\
& =\frac{1}{n-1}(X \wedge \delta \psi(J Y, Z)+X \wedge \delta \psi(Y, J Z)) \\
& =-\frac{1}{n-1}(\omega \wedge J \delta \psi(X, J Y, Z)+\omega \wedge J \delta \psi(X, Y, J Z)) .
\end{aligned}
$$

It follows that $\frac{1}{3} d \psi+\frac{1}{n-1} \omega \wedge J \delta \psi$ is a (real) $J$-invariant 3 -form, and so it must be zero - for instance one can use the identity

$$
\begin{aligned}
2 \beta(J X, Y, Z)= & \beta(Z, J X, Y)+\beta(Z, X, J Y) \\
& -\beta(X, J Y, Z)-\beta(X, Y, J Z) \\
& +\beta(Y, J Z, X)+\beta(Y, Z, J X) .
\end{aligned}
$$

Equation (85) follows immediately. Next, the defining equation (84) implies

$(n-1)\left(\nabla_{X} \psi(J Y)+\nabla_{Y} \psi(J X)\right)=\delta \psi(X) J Y+\delta \psi(Y) J X-2\langle X, Y\rangle J \delta \psi$.

Taking the trace of this formula as a function of $Y$ gives

$$
(n-1)(2 d \operatorname{tr} \psi(X)+J \delta \psi(X))=3 J \delta \psi(X),
$$

which is manifestly equivalent to (86). 
(iii) This is a simple verification using (85):

$$
\begin{aligned}
\nabla_{X} \psi-\frac{1}{n-1} d f(X) \omega & =\frac{1}{n-1}\left(-X \wedge J d f+\iota_{X}(\omega \wedge d f)-d f(X) \omega\right) \\
& =-\frac{1}{n-1}\left(d f \wedge J X-d^{c} f \wedge X\right) .
\end{aligned}
$$

Note that (86) gives $d \operatorname{tr} \phi=d \operatorname{tr} \psi-\frac{m}{n-1} d f=-\frac{2}{n-1} d f$.

q.e.d.

We remark that there is also a connection between hamiltonian 2forms and Killing tensors (cf. [16]), i.e., symmetric 2-tensors $S$ satisfying sym $\nabla S=0$. Indeed $\phi$ is hamiltonian if and only if $A=\phi+\sigma \omega$ is closed and $S=J(\phi-\sigma \omega)$ is a Killing tensor. For the reverse implication, observe that $d A=0$ determines $\nabla_{X} \phi(Y, Z)+\nabla_{Y} \phi(X, Z)+\nabla_{Z} \phi(X, Y)$ in terms of the 1-form $d \sigma$, while sym $\nabla S=0$ determines $\nabla_{X} \phi(J Y, Z)+$ $\nabla_{Y} \phi(J Z, X)+\nabla_{Z} \phi(J X, Y)$. Replacing $J Y$ by $Y$ in the second expression and adding to the first yields a formula for $2 \nabla_{X} \phi(Y, Z)+$ $\nabla_{Y} \phi(Z, X)-\nabla_{J Y} \phi(J Z, X)$ and the $J$-invariant part (in $Y, Z$ ) is (12).

\section{Appendix B. Vandermonde matrices}

B.1. The inverse of a Vandermonde matrix. A Vandermonde matrix is a $(m \times m)$-matrix of the form

$$
V=V\left(\xi_{1}, \ldots \xi_{m}\right)=\left[\begin{array}{ccc}
\xi_{1}^{m-1} & \cdots & \xi_{m}^{m-1} \\
-\xi_{1}^{m-2} & \cdots & -\xi_{m}^{m-2} \\
\vdots & \cdots & \vdots \\
(-1)^{m-1} & \cdots & (-1)^{m-1}
\end{array}\right]
$$

where the $\xi_{j}$ 's are $m$ independent variables; the entries of the Vandermonde matrix $V$ are thus defined by $V_{r j}=(-1)^{r-1} \xi_{j}^{m-r}$.

We denote by $\sigma_{r}$ the elementary symmetric functions of the $\xi_{j}$ 's, so that

$$
\prod_{j=1}^{m}\left(t-\xi_{j}\right)=t^{m}-\sigma_{1} t^{m-1}+\cdots+(-1)^{m} \sigma_{m},
$$

for any $t$. We also define $\sigma_{0}=1$.

Removing the variable $\xi_{j}$ (equivalently, differentiating with respect to $\xi_{j}$ ) gives

$$
\prod_{k \neq j}\left(t-\xi_{k}\right)=t^{m-1}-\sigma_{1}\left(\hat{\xi}_{j}\right) t^{m-1}+\ldots+(-1)^{m-1} \sigma_{m-1}\left(\hat{\xi}_{j}\right),
$$

where the $\sigma_{r}\left(\hat{\xi}_{j}\right)$ are the elementary symmetric functions of the $m-1$ variables $\xi_{1}, \ldots \hat{\xi}_{j}, \ldots \xi_{m}$ ( $\xi_{j}$ deleted). By putting $t=\xi_{i}$ in the above identity, we get

$$
\xi_{i}^{m-1}-\sigma_{1}\left(\hat{\xi}_{j}\right) \xi_{i}^{m-2}+\cdots+(-1)^{m-1} \sigma_{m-1}\left(\hat{\xi}_{j}\right)=\Delta_{j} \delta_{i j},
$$


where $\delta_{i j}$ is the Kronecker symbol and $\Delta_{j}=\prod_{k \neq j}\left(\xi_{j}-\xi_{k}\right)$.

This means that the matrix $W$ whose entries are $W_{i r}=\sigma_{r-1}\left(\hat{\xi}_{i}\right) / \Delta_{i}$, i.e.,

is a left-inverse of $V$ :

$$
W=\left[\begin{array}{cccc}
\frac{1}{\Delta_{1}} & \frac{\sigma_{1}\left(\hat{\xi}_{1}\right)}{\Delta_{1}} & \ldots & \frac{\sigma_{m-1}\left(\hat{\xi}_{1}\right)}{\Delta_{1}} \\
\vdots & \vdots & \ldots & \vdots \\
\frac{1}{\Delta_{m}} & \frac{\sigma_{1}\left(\hat{\xi}_{m}\right)}{\Delta_{m}} & \cdots & \frac{\sigma_{m-1}\left(\hat{\xi}_{m}\right)}{\Delta_{m}}
\end{array}\right],
$$

$$
\sum_{r=1}^{m} W_{i r} V_{r j}=\delta_{i j}
$$

B.2. The determinant of a Vandermonde matrix. In order to compute the determinant $\operatorname{det} V$ of $V$, we use the fact that $W_{11}=1 / \Delta_{1}$ is equal to the determinant of the minor of $V_{11}$ in $V$ divided by $\operatorname{det} V$. Now the minor of $V_{11}$ is clearly $-V\left(\xi_{2}, \ldots, \xi_{m}\right)$; we thus get the following induction formula:

$$
\operatorname{det} V\left(\xi_{1}, \ldots, \xi_{m}\right)=(-1)^{m-1}\left(\xi_{1}-\xi_{2}\right) \ldots\left(\xi_{1}-\xi_{m}\right) \operatorname{det} V\left(\xi_{2}, \ldots, \xi_{m}\right),
$$

from which we readily infer that

$$
\operatorname{det} V=(-1)^{m(m-1) / 2} \prod_{i<j}\left(\xi_{i}-\xi_{j}\right) .
$$

Notice that we also have

$$
(\operatorname{det} V)^{2}=(-1)^{m(m-1) / 2} \prod_{j=1}^{m} \Delta_{j} .
$$

Indeed, both sides are products of elements of the form $\xi_{i}-\xi_{j}$ : for each $i<j$, we get $\left(\xi_{i}-\xi_{j}\right)^{2}$ in the left hand side, and $\left(\xi_{i}-\xi_{j}\right)\left(\xi_{j}-\xi_{i}\right)=$ $-\left(\xi_{i}-\xi_{j}\right)^{2}$ in the right hand side.

B.3. Vandermonde identities. In the ring of $m \times m$ matrices, a left inverse is also a right-inverse, so that:

$$
\sum_{j=1}^{m} V_{s j} W_{j r}=\delta_{r s}
$$

we thus obtain the following Vandermonde identity

$$
\sum_{j=1}^{m} \frac{(-1)^{s-1} \xi_{j}^{m-s} \sigma_{r-1}\left(\hat{\xi}_{j}\right)}{\Delta_{j}}=\delta_{r s}
$$

for any pair $r, s=1, \ldots m$. In particular, with $r=1$, we have that

$$
\sum_{j=1}^{m} \frac{\xi_{j}^{m-s}}{\Delta_{j}}=\delta_{s 1}
$$


for $s=1, \ldots m$. This identity for $s=1$ may be extended to all $s \leq 1$ to give

$$
\sum_{j=1}^{m} \frac{\xi_{j}^{m-1+p}}{\Delta_{j}}=h_{p}
$$

for all $p \geq 0$, where $h_{p}$ is the $p$ th complete symmetric function of $\xi_{1}, \ldots \xi_{m}$. By multiplying by $t^{p}$, for a formal variable $t$, and summing over $p \geq 0$, this equation may be rewritten

$$
\sum_{j=1}^{m} \frac{\xi_{j}^{m-1}}{\left(1-\xi_{j} t\right) \Delta_{j}}=\prod_{k=1}^{m} \frac{1}{1-\xi_{k} t}
$$

where the right hand side denotes the (formal) product of geometric series. Hence, to prove (95), it suffices to observe that

$$
\sum_{j=1}^{m} \xi_{j}^{m-1} \prod_{k \neq j} \frac{1-\xi_{k} t}{\xi_{j}-\xi_{k}}=1
$$

This follows because the left hand side is a polynomial in $t$, of degree at most $m-1$, whose value at $t=1 / \xi_{j}$ is equal to 1 for all $j=1, \ldots m$. (In fact, this is more or less the Lagrange interpolation formula.)

Similarly, we can extend (93) to obtain

$$
\sum_{j=1}^{m} \frac{\xi_{j}^{m+k} \sigma_{r-1}\left(\hat{\xi}_{j}\right)}{\Delta_{j}}=\sum_{s=0}^{k}(-1)^{s} h_{k-s} \sigma_{r+s}
$$

for all $r=1, \ldots m$ and all $k \geq 0$. Here, by convention, $\sigma_{r+s}=0$ for $r+s>m$. We reduce (96) to (95) by means of the obvious identity:

$$
\xi_{j}^{m+k} \sigma_{r-1}\left(\hat{\xi}_{j}\right)=\sum_{s=0}^{m-r}(-1)^{s} \xi_{j}^{m-1+k-s} \sigma_{r+s} .
$$

(Evidently $\xi_{j} \sigma_{r-1}\left(\hat{\xi}_{j}\right)=\sigma_{r}-\sigma_{r}\left(\hat{\xi}_{j}\right)$.) Substitute this into the left hand side of (96), and note that the summation over $s$ can be made from 0 to $k$, using the Vandermonde identity (94) to eliminate any extra terms. Now applying (95) for each $s$ yields the right hand side of (96).

There is one further identity we shall need, namely

$$
\frac{\partial}{\partial \xi_{i}}\left(\sum_{j=1}^{m} \frac{\xi_{j}^{m+k} \sigma_{r-1}\left(\hat{\xi}_{j}\right)}{\Delta_{j}}\right)=\sigma_{r-1}\left(\hat{\xi}_{i}\right) \sum_{s=0}^{k} h_{k-s} \xi_{i}^{s} .
$$

We prove this using (96): multiplying by $t^{k}$ and summing over $k$, it suffices to show

$$
\sum_{s \geq 0} \frac{\partial}{\partial \xi_{i}}\left(\frac{(-1)^{s} \sigma_{r+s} t^{s}}{\prod_{j=1}^{m}\left(1-\xi_{j} t\right)}\right)=\frac{\sigma_{r-1}\left(\hat{\xi}_{i}\right)}{\left(1-\xi_{i} t\right) \prod_{j=1}^{m}\left(1-\xi_{j} t\right)} .
$$


This holds since direct computation of the left hand side gives

$$
\sum_{s \geq 0} \frac{(-1)^{s} \sigma_{r+s-1}\left(\hat{\xi}_{i}\right) t^{s}+(-1)^{s} \sigma_{r+s}\left(\hat{\xi}_{i}\right) t^{s+1}}{\left(1-\xi_{i} t\right) \prod_{j=1}^{m}\left(1-\xi_{j} t\right)}
$$

using $\sigma_{r+s}\left(\hat{\xi}_{i}\right)=\sigma_{r+s}-\xi_{i} \sigma_{r+s-1}\left(\hat{\xi}_{i}\right)$. All terms now cancel in pairs except the first one with $s=0$.

In fact we shall only make serious use of the identities (96) and (97) for $0 \leq k \leq 2$. In particular (96) implies

$$
\begin{aligned}
\sum_{j=1}^{m} \frac{\xi_{j}^{m}}{\Delta_{j}} \sigma_{r-1}\left(\hat{\xi}_{j}\right) & =\sigma_{r}, \\
\sum_{j=1}^{m} \frac{\xi_{j}^{m+1}}{\Delta_{j}} \sigma_{r-1}\left(\hat{\xi}_{j}\right) & =\sigma_{1} \sigma_{r}-\sigma_{r+1}, \\
\sum_{j=1}^{m} \frac{\xi_{j}^{m+2}}{\Delta_{j}} \sigma_{r-1}\left(\hat{\xi}_{j}\right) & =\left(\sigma_{1}^{2}-\sigma_{2}\right) \sigma_{r}-\sigma_{1} \sigma_{r+1}+\sigma_{r+2} .
\end{aligned}
$$

\section{References}

[1] M. Abreu, Kähler geometry of toric varieties and extremal metrics, Internat. J. Math. 9 (1998) 641-651, MR 1644291, Zbl 0932.53043.

[2] V. Apostolov \& P. Gauduchon, Self-dual Einstein Hermitian 4-manifolds, Ann. Scuola Norm. Sup. Pisa (5) 1 (2002) 203-243, MR 1994808.

[3] V. Apostolov, D.M.J. Calderbank, \& P. Gauduchon, The geometry of weakly self-dual Kähler surfaces, Compositio Math. 135 (2003) 279-322, MR 1956815, Zbl 1031.53045 .

[4] V. Apostolov, D.M.J. Calderbank, C.W. Tonnesen-Friedman, \& P.Gauduchon, Hamiltonian 2-forms in Kähler geometry II: Global classification, J. Differential Geometry 68 (2004) 277-345, MR 2144249.

[5] _ Hamiltonian 2-forms in Kähler geometry III: Extremal metrics and stability, available at math.DG/0511118.

[6] _ Hamiltonian 2-forms in Kähler geometry IV: Weakly Bochner-flat Kahler manifolds, available at math.DG//0511119.

[7] A.L. Besse, Einstein manifolds, Ergeb. Math. Grenzgeb., 3, Springer-Verlag, Berlin, Heidelberg, New York, 1987, MR 0867684, Zbl 0613.53001.

[8] R. Bryant, Bochner-Kähler metrics, J. Amer. Math. Soc. 14 (2001) 623-715, MR 1822355, Zbl 1006.53019. 
[9] E. Calabi, Métriques kählériennes et fibrés holomorphes, Ann. Sci. Ecole Norm. Sup. (4) 12 (1979) 269-294, MR 0543218, Zbl 0431.53056.

[10] Extremal Kähler metrics, Seminar on Differential Geometry, 259290, Ann. of Math. Stud., 102, Princeton Univ. Press, Princeton, NJ, 1982, MR 0645743, Zbl 0487.53057.

[11] A. Derdziński \& G. Maschler, Local classification of conformally-Einstein Kähler metrics in higher dimensions, Proc. London Math. Soc. (3) 87 (2003), 779-819, MR 2005883.

[12] J.J. Duistermaat \& G.J. Heckman, On the variation in the cohomology of the symplectic form of the reduced phase space, Invent. Math. 69 (1982) 259-268, MR 0674406, Zbl 0503.58015.

[13] A. Gray, Pseudo-Riemannian almost product manifolds and submersions, J. Math. Mech. 16 (1967) 715-737, MR 0205184, Zbl 0147.21201.

[14] V. Guillemin, Kähler structures on toric varieties, J. Differential Geom. 40 (1994) 285-309, MR 1293656, Zbl 0813.53042.

[15] A.D. Hwang \& M.A. Singer, A momentum construction for circle-invariant Kahler metrics, Trans. Amer. Math. Soc. 354 (2002) 2285-2325, MR 1885653, Zbl 0987.53032 .

[16] W. Jelonek, Compact Kähler surfaces with harmonic anti-self-dual Weyl tensor, Diff. Geom. Appl. 16 (2002), 267-276, MR 1900748, Zbl 1037.53028.

[17] N. Koiso \& Y. Sakane, Nonhomogeneous Kähler-Einstein metrics on compact complex manifolds, in 'Curvature and Topology of Riemannian Manifolds' (Kataka, 1985), Lecture Notes in Math., 1201, Springer-Verlag, Berlin, 1986, 165-179, MR 0859583, Zbl 0591.53056.

[18] B. Kostant, Holonomy and the Lie algebra of infinitesimal motions of a Riemann manifold, Trans. Amer. Math. Soc. 80 (1955) 528-542, MR 0084825, Zbl 0066.16001.

[19] C.R. LeBrun, Explicit self-dual metrics on $\mathbb{C} P^{2} \# \cdots \# \mathbb{C} P^{2}$, J. Differential Geom. 34 (1991) 223-253, MR 1114461, Zbl 0725.53067.

[20] A. Moroianu and U. Semmelmann, Twistor forms on Kähler manifolds, Ann. Sc. Norm. Super. Pisa Cl. Sci. (5) 2 (2003) 823-845, MR 2040645.

[21] B. O'Neill, The fundamental equations of a submersion, Michigan Math. J. 13 (1966) 459-469, MR 0200865, Zbl 0145.18602.

[22] H. Pedersen \& Y.S. Poon, Hamiltonian construction of Kähler-Einstein metrics and Kähler metrics of constant scalar curvature, Comm. Math. Phys. 136 (1991) 309-326, MR 1096118, Zbl 0792.53065.

[23] M. Sekizawa, On conformal Killing tensors of degree 2 in Kählerian spaces, TRU Math. 6 (1970) 1-5, MR 0367850, Zbl 0238.53017.

[24] U. Semmelmann, Conformal Killing forms on Riemannian manifolds, Habilitationschrift, Universität München, 2002, available at arXiv:math.DG/0206117.

[25] C. Tønnesen-Friedman, Extremal Kähler metrics on minimal ruled surfaces, J. Reine Angew. Math. 502 (1998) 175-197, MR 1647571, Zbl 0921.53033.

[26] Extremal Kahler metrics and Hamiltonian functions, II, Glasg. Math. J. 44 (2002) 241-253, MR 0837617, Zbl 0602.57014. 
[27] M.Y. Wang, Einstein metrics from symmetry and bundle constructions, Surv. Diff. Geom., VI, Int. Press, Boston, MA, 1999, 287-325, MR 1798614, Zbl 1003.53037.

DÉPARTEMEnt DE MATHÉmatiques UQAM, C.P. 8888

SucC. Centre-Ville Montréal (QuÉBEC) H3C 3P8 CANADA

E-mail address: apostolo@math.uqam.ca

SCHOOL OF MATHEMATics

UNIVERSITY OF EDINBURGH

King's Buildings, MAYField RoAd

EDINBURGH EH9 3JZ SCOTLAND

E-mail address: davidmjc@maths.ed.ac.uk

Current address

Department of Mathematics

University of York

Heslington, York

YO10 5DD, England

E-mail address: dc511@york.ac.uk

Centre de Mathématiques

Ecole Polytechnique

UMR 7640 DU CNRS

91128 Palaiseau, France

E-mail address: pg@math.polytechnique.fr 